\title{
Local order in Cr-Fe-Co-Ni: Experiment and electronic structure calculations
}

\author{
B. Schönfeld, ${ }^{1}$ C. R. Sax, ${ }^{1}$ J. Zemp, ${ }^{1}$ M. Engelke,${ }^{2}$ P. Boesecke, ${ }^{3}$ T. Kresse,${ }^{4}$ T. Boll,${ }^{5}$ T. Al-Kassab,${ }^{4}$ \\ O. E. Peil, ${ }^{6}$ and A. V. Ruban ${ }^{6,7}$ \\ ${ }^{1}$ Laboratory of Metal Physics and Technology, Department of Materials, ETH Zurich, 8093 Zurich, Switzerland \\ ${ }^{2}$ Department of Radiation Safety and Security, Paul Scherrer Institute, 5232 Villigen PSI, Switzerland \\ ${ }^{3}$ ESRF - The European Synchrotron, 38043 Grenoble Cedex 9, France \\ ${ }^{4}$ King Abdullah University of Science and Technology, Division of Physical Sciences, Saudi Arabia \\ ${ }^{5}$ Institute for Applied Materials IAM-WK and Karlsruhe Nano Micro Facility, Karlsruhe Institute of Technology, \\ 76344 Eggenstein-Leopoldshafen, Germany \\ ${ }^{6}$ Materials Center Leoben Forschung GmbH, 8700 Leoben, Austria \\ ${ }^{7}$ Department of Materials Science and Engineering, Royal Institute of Technology, 10044 Stockholm, Sweden
}

(Received 21 October 2018; revised manuscript received 18 December 2018; published 18 January 2019)

\begin{abstract}
A quenched-in state of thermal equilibrium (at $723 \mathrm{~K}$ ) in a single crystal of $\mathrm{Cr}$-Fe-Co-Ni close to equal atomic percent was studied. Atom probe tomography revealed a single-phase state with no signs of long-range order. The presence of short-range order (SRO) was established by diffuse x-ray scattering exploiting the variation in scattering contrast close to the absorption edges of the constituents: At the incoming photon energies of 5969, 7092, and $8313 \mathrm{eV}$, SRO maxima that result from the linear superposition of the six partial SRO scattering patterns, were always found at $X$ position. Electronic structure calculations showed that this type of maximum stems from the strong $\mathrm{Cr}-\mathrm{Ni}$ and $\mathrm{Cr}$-Co pair correlations, that are furthermore connected with the largest scattering contrast at $5969 \mathrm{eV}$. The calculated effective pair interaction parameters revealed an order-disorder transition at approximately $500 \mathrm{~K}$ to a $L 1_{2}$-type $(\mathrm{Fe}, \mathrm{Co}, \mathrm{Ni})_{3} \mathrm{Cr}$ structure. The calculated magnetic exchange interactions were dominantly of the antiferromagnetic type between $\mathrm{Cr}$ and any other alloy component and ferromagnetic between $\mathrm{Fe}, \mathrm{Co}$, and Ni. They yielded a Curie temperature $\left(T_{\mathrm{C}}\right)$ of $120 \mathrm{~K}$, close to experimental findings. Despite the low value of $T_{\mathrm{C}}$, the global magnetic state strongly affects chemical and elastic interactions in this system. In particular, it significantly increases the ordering tendency in the ferromagnetic state compared to the paramagnetic one.
\end{abstract}

DOI: 10.1103/PhysRevB.99.014206

\section{INTRODUCTION}

Diffuse scattering using $\mathrm{x}$ rays and neutrons has turned up to be an invaluable tool to characterize the microstructure of alloys. When states of thermal equilibrium were investigated, electronic structure calculations were an indispensable tool to further address the reliability of these microstructural data by criteria that go beyond a comparison of order-disorder transition temperatures obtainable from direct observations. Such investigations by diffuse scattering required the use of single crystalline material. However, these studies were mostly restricted to binary systems [1-5].

Ternary alloys were rarely investigated (see, e.g., Refs. [6-9]). With neutron scattering, isotopic replacement then gets mandatory. In the case of $\mathrm{x}$-ray scattering, measurements close to the absorption edges of the sample constituents are required. Still, the stability in extracting data remains a severe problem, in addition to the much prolonged measuring time. There is also the problem that different samples may differ in composition and thermal history.

As a real-space approach to the three-dimensional (3D) atomic arrangement in alloys, atom probe tomography (APT) $[10,11]$ has attracted much interest. However, the missing registration of ions due to the limited detector efficiency as well as the spatial uncertainty in reconstructing the actual position of atoms in the tip, impose limitations. On one side, heterogeneity on a nanoscale can be well resolved. Also, preferential sublattice occupation in long-range ordered alloys was characterized [12]. On the other side, the quantification of short-range order (SRO) still presents a challenge (see, e.g., Ref. [13]). Of course there is the advantage that more correlation functions can be obtained from APT (between atoms of any species or even between sets of species) than is possible in diffuse scattering where SRO parameter sets are determined for pairs of atoms belonging to different species. A detailed study on local order in Fe-rich Fe-Al showed that SRO parameter sets from APT clearly underestimate the correlations: A state rather close to an uncorrelated configuration was found, in contrast to what is known from diffuse $\mathrm{x}$-ray and neutron scattering [13].

More recently, the so-called high-entropy alloys (HEAs) have attracted much interest [14-16]. These alloys are also repeatedly termed multiprincipal element alloys (MPEAs) with at least four or five elements in equal or near-equal fraction. Four items were claimed as typical features of such alloys: (i) They often form solid solutions at ambient temperature due to high constitutional entropy. (ii) Kinetics is sluggish in comparison to the case of substitutional impurities in the respective binaries. (iii) They show strong lattice distortions when elements of different sizes are principal elements, 
presenting the reason for favorable strength. (iv) Mechanical properties are quite tunable by changes in constituents and their fractions (so-called cocktail effect). As stressed by Miracle and Senkov [16], items (i) to (iii) are hypotheses and detailed experimental support is required. For instance, the situation of entropic stabilization of a solid solution together with severe lattice distortions requires further clarification. Here, another reason for alloy strengthening might have been occasionally overlooked: the presence of small precipitates may remain unnoted in standard $\mathrm{x}$-ray powder diffraction. The need for accompanying high-resolution studies using transmission electron microscopy (TEM) or APT was repeatedly stressed $[15,17]$.

A large group of HEAs hitherto studied contains $\mathrm{Cr}$, $\mathrm{Fe}$, $\mathrm{Co}$, and $\mathrm{Ni}$ as principal elements, beside elements like $\mathrm{Al}$, $\mathrm{Cu}, \mathrm{Ti}$, and $\mathrm{Mn}$. Reports differ whether the alloy $\mathrm{Cr}-\mathrm{Fe}-$ $\mathrm{Co}-\mathrm{Ni}$ in equal atomic fraction is a single-phase (presenting an fcc solid solution) or a two-phase state (see Ref. [17] and references therein). No signs of long-range order were detected by x-ray and neutron measurements using polycrystalline material by Lucas et al. [18]. Here, samples were slowly cooled from $1273 \mathrm{~K}$ to RT, with an intermediate stop for 2 weeks at $753 \mathrm{~K}$. X-ray scattering was done using the $K$-edge energies of the four elements as incoming photon energies. However, a huge unwanted scattering contribution due to the presence of resonant Raman scattering/fluorescence is then present that might hide subtle scattering modulations due to local order (in addition to the use of polycrystalline material)

For the ternary system $\mathrm{Fe}-21$ at.\% Cr- 23 at.\% Ni, Cénédèse et al. [6] performed a detailed study using isotopic replacement and diffuse neutron scattering. They employed single crystals, mandatory in detailed SRO studies. Samples were aged for $10 \mathrm{~h}$ at $773 \mathrm{~K}$ where an increase in residual resistivity and thermoelectric power had been stated. Neglecting static atomic displacements in data evaluation, they found that scattering due to local order leads to a diffuse maximum at $X$ position for Ni-Cr pairs, $W$ position for $\mathrm{Fe}-\mathrm{Cr}$ pairs, and $\Gamma$ position for Fe-Ni pairs. Subsequent electronic structure calculations by Ruban and Dehghani [19] yielded quite comparable results: diffuse maxima at $X$ position for $\mathrm{Ni}-\mathrm{Cr}$ and $\mathrm{Fe}-\mathrm{Cr}$ pairs, at $\Gamma$ position for Fe-Ni pairs. Experimental and calculated data both showed the largest intensity modulations due to Ni-Cr pairs. Interestingly and in contrast to the binary $\mathrm{Ni}-\mathrm{Cr}$ system, a diffuse maximum in local order is no longer found at $W$ position. This location was well established for $\mathrm{Ni}-11(20,25,33)$ at. $\% \mathrm{Cr}$ by diffuse $\mathrm{x}$-ray and neutron scattering [20-23] and confirmed by a recent first-principles study [24].

Singh et al. [25] have recently calculated effective interactions and ordering in $\mathrm{Al}-\mathrm{Co}-\mathrm{Cr}-\mathrm{Fe}-\mathrm{Ni}$ alloys with compositions close to the equimolar one. They found that the strongest interactions driving $L 1_{2}$ ordering are $\mathrm{Cr}-\mathrm{Al}$ and $\mathrm{Cr}$-Co ones. Since Al strongly interacts with the other alloy components, the direct use of the results of this work for the $\mathrm{Cr}-\mathrm{Fe}$ $\mathrm{Co}-\mathrm{Ni}$ system is problematic. A quite pronounced tendency towards the $\mathrm{L1}_{2}-(\mathrm{Fe}, \mathrm{Co}, \mathrm{Ni})_{3} \mathrm{Cr}$ type of ordering in $\mathrm{Cr}-\mathrm{Fe}-\mathrm{Co}-$ $\mathrm{Ni}$ has been also theoretically predicted in Refs. [26,27] in direct first-principles modeling of the corresponding structure. However, these investigations do not provide information about effective interactions which can be used in statistical thermodynamics simulations.

The intention of this work was to study local order in the quaternary $\mathrm{Cr}-\mathrm{Fe}-\mathrm{Co}-\mathrm{Ni}$ close to equal atomic percent. Diffuse x-ray scattering using the tunability of synchrotron radiation together with high-resolution real-space information by APT is employed (results in Secs. V and VI, following basics in Secs. II-IV). As SRO scattering always comprises a linear superposition of six pair correlation functions (hardly separable), electronic structure calculations are required to provide information about effective interactions and further details on the microstructure through statistical thermodynamics modeling (Sec. VII). This is possible as a state of thermal equilibrium is under study. Based on the chemical interactions, the order-disorder transition temperature together with the ordered fcc structure will be determined. Using the calculated magnetic interactions, the Curie temperature will also be determined and compared to experimental findings. The combination of these approaches offers the opportunity to find out whether such a multicomponent alloy exhibits any new microstructural feature (Secs. VIII and IX).

\section{METHODOLOGY}

\section{A. Basics of elastic diffuse scattering}

Diffuse x-ray scattering from a multicomponent alloy comprises the elastic scattering contribution due to atomic short-range order (SRO) and static atomic displacements that is often approximately treated in terms of size-effect (SE) and Huang $(\mathrm{H})$ scattering (see Refs. [1-5]). The inelastic scattering contributions are fluorescence and resonant Raman scattering (RRS), Compton scattering, and thermal diffuse scattering (TDS). The quantity of central interest in this work is local order. For the present quaternary system, there are six partial SRO parameter sets $\alpha_{l m n}^{i j}$ defined as in the case of binary fcc alloys [28]. Here $i, j=1$ to $4(\mathrm{Cr}, \mathrm{Fe}, \mathrm{Co}, \mathrm{Ni})$ with $i \neq j$ marks the component and $l m n$ the neighboring shell. The Fourier transformation of these $\alpha_{l m n}^{i j}$ are the partial SRO scattering intensities [29]

$$
I_{\mathrm{SRO}}^{i j}(\mathbf{h})=\sum_{l m n} \alpha_{l m n}^{i j} \cos \left(\pi h_{x} l\right) \cos \left(\pi h_{y} m\right) \cos \left(\pi h_{z} n\right),
$$

with scattering vector $\mathbf{h}=\left(h_{x}, h_{y}, h_{z}\right)$ in reciprocal lattice units (rlu). In Laue units (Lu, $1 \mathrm{Lu}=\sum_{i} \sum_{j>i} c_{i} c_{j}\left|f_{i}-f_{j}\right|^{2}$ with $c_{i}=$ atomic fraction and $f_{i}=$ atomic scattering factor of component $i$ ), the total SRO scattering is then

$$
\begin{gathered}
I_{\mathrm{SRO}}(\mathbf{h})=\sum_{l m n} \alpha_{l m n}^{\mathrm{eff}} \cos \left(\pi h_{x} l\right) \cos \left(\pi h_{y} m\right) \cos \left(\pi h_{z} n\right), \\
\alpha_{l m n}^{\mathrm{eff}}=\frac{\sum_{i} \sum_{j>i} c_{i} c_{j}\left|f_{i}-f_{j}\right|^{2} \alpha_{l m n}^{i j}}{\sum_{i} \sum_{j>i} c_{i} c_{j}\left|f_{i}-f_{j}\right|^{2}} .
\end{gathered}
$$

In contrast to any partial SRO scattering $I_{\mathrm{SRO}}^{i j}(\mathbf{h})$, the total SRO scattering $I_{\text {SRO }}(\mathbf{h})$ must be non-negative for any $\mathbf{h}$ (see Refs. [30,31]). For a homogeneous solid solution $\left(\alpha_{l m n \neq 000}^{i j}=\right.$ 0 ) monotonic Laue scattering is present.

Size effect scattering $I_{\mathrm{SE}}(\mathbf{h})$ of the present quaternary system comprises 27 terms (considering that there has to be 
an average cubic lattice). Neglecting any species dependence, one obtains an expression of the form

$$
I_{\mathrm{SE}}(\mathbf{h})=\mathbf{h} \cdot \mathbf{Q}(\mathbf{h}),
$$

with, e.g.,

$$
Q_{x}(\mathbf{h})=\sum_{l m n} \gamma_{l m n}^{x, \mathrm{eff}} \sin \left(\pi h_{x} l\right) \cos \left(\pi h_{y} m\right) \cos \left(\pi h_{z} n\right),
$$

where $\gamma_{l m n}^{x, \text { eff }}$, e.g., is a linear superposition of the $10-1$ static atomic pair displacement parameters along $x$.

The separation of elastic scattering into the species independent terms of SRO and SE scattering presents an approximation as the $|\mathbf{h}|$ dependence of the atomic scattering factors $f_{i}$ differs among the elements. Still, good agreement in $\gamma_{l m n}^{x \text {,eff }}$ was seen between both approaches (cf. Refs. [32,33]) in the case of binary alloys. Any such separation scheme is beyond the scope of this study, due to the limited range explored in reciprocal space and the limited number of selected energies. Instead, $\alpha_{l m n}^{\text {eff }}$ and $\gamma_{l m n}^{x \text {,eff }}$ were determined by least-squares fitting and then compared with results from electronic structure calculations.

\section{B. Considerations to statistical thermodynamics}

The configurational state of alloys was studied using the following Hamiltonian:

$$
\begin{aligned}
H= & \frac{1}{2} \sum_{p} \sum_{\alpha, \beta \neq \delta} V_{p}^{(2)-\alpha \beta[\delta]} \sum_{i j \in p} \delta c_{i}^{\alpha} \delta c_{j}^{\beta} \\
& +\frac{1}{3} \sum_{t} \sum_{\alpha, \beta, \gamma \neq \delta} V_{t}^{(3)-\alpha \beta \gamma[\delta]} \sum_{i, j, k} \delta c_{i}^{\alpha} \delta c_{j}^{\beta} \delta c_{k}^{\gamma}+\text { h.o.t. }
\end{aligned}
$$

The summation is performed over different types of clusters ( $p$ and $t$ stand for indexes of the pairs and triangles), alloy components (designated by Greek letters), and lattice sites $(i, j$, and $k) ; V_{p}^{(2)-\alpha \beta[\delta]}$ and $V_{t}^{(3)-\alpha \beta \gamma[\delta]}$ are the pair- and three-site effective concentration, magnetic state, and volume dependent interactions; and $\delta c_{i}^{\alpha}=c_{i}^{\alpha}-c^{\alpha}$ is the concentration fluctuation of the $\alpha$ component at site $i$ from its average concentration in the alloy $c^{\alpha}$.

To analyze the ordering behavior in a multicomponent system, it is very convenient to reduce the dominant contribution from pair interactions in Eq. (6) to a quasibinary form [19]

$$
H^{(2)}=-\frac{1}{2} \sum_{p} \sum_{\alpha \neq \beta} \widetilde{V}_{p}^{(2)-\alpha \beta} \sum_{i j \in p} \delta c_{i}^{\alpha} \delta c_{j}^{\beta},
$$

where $\widetilde{V}_{p}^{(2)-\alpha \beta}$ are the usual binary effective interactions describing the mutual ordering of $\alpha$ and $\beta$ atoms and are related to the multicomponent effective pair interactions $V_{p}^{(2)-\alpha \beta[\delta]}$ as $[19,34,35]$

$$
V_{p}^{(2)-\alpha \beta[\delta]}=\frac{1}{2}\left[\widetilde{V}_{p}^{(2)-\alpha \delta}+\widetilde{V}_{p}^{(2)-\beta \delta}-\widetilde{V}_{p}^{(2)-\alpha \beta}\right] .
$$

In contrast to $\widetilde{V}_{p}^{(2)-\alpha \beta}$ interactions, whose positive values mean the attraction of $\alpha$ and $\beta$ species at the $p$ th coordination shell, mixed type interactions $V_{p}^{(2)-\alpha \beta[\delta]}$ do not have a simple interpretation.

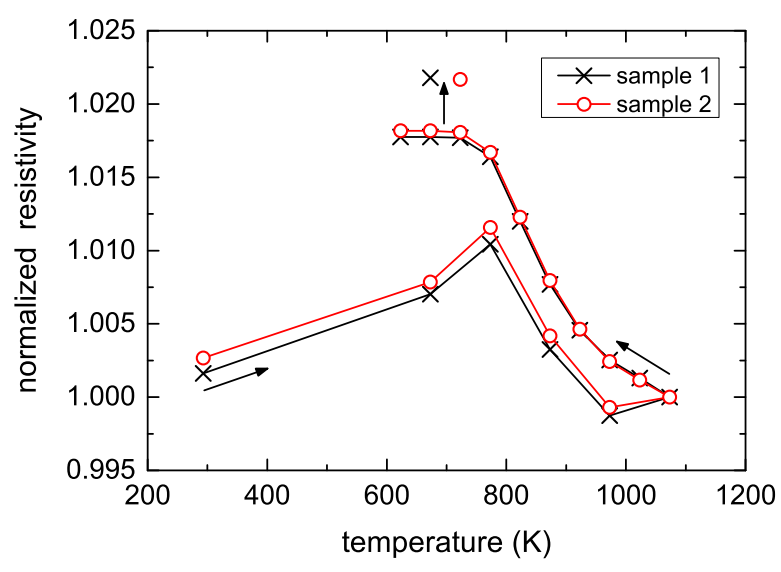

FIG. 1. Normalized resistivity from $\mathrm{Cr}-\mathrm{Fe}-\mathrm{Co}-\mathrm{Ni}$ versus aging temperature for two samples. At the end of the aging path RT (as-grown state) $\rightarrow 1073 \mathrm{~K} \rightarrow 623 \mathrm{~K}$ (holding time of $24 \mathrm{~h}$ for any aging temperature), sample 1 was additionally heat treated at $673 \mathrm{~K}$ for 119 days, sample 2 at $723 \mathrm{~K}$ for 16 days.

\section{EXPERIMENT}

The starting materials were 99.99 pure $\mathrm{Cr}, 99.95$ at.\% pure $\mathrm{Fe}, 99.99$ at.\% pure Ni from MRC (Toulouse, France) and 99.95 at.\% pure Co from Alfa Aesar. A single crystal was grown by the Bridgman technique in an $\mathrm{Al}_{2} \mathrm{O}_{3}$ crucible aiming for an equiatomic fraction in $\mathrm{Cr}, \mathrm{Fe}, \mathrm{Co}$, and $\mathrm{Ni}$. By adapting a sufficiently slow growing speed, a dendritic growth was avoided as checked metallographically.

For the APT investigations, pieces with a [001] orientation were cut by wire saw and mechanically polished, being finally $0.2 \times 0.2 \times 10 \mathrm{~mm}^{3}$ in dimension. Tips were prepared in a solution of perchloric acid with 15 vol. \% acetic acid and a dc voltage of 5-15 V to shape tips with curvature radii below $100 \mathrm{~nm}$. Tips were resharpened in a focused ion beam system (FEI Quanta 3D FEG) using acceleration voltages from 15 down to $5 \mathrm{kV}$ and low beam currents to reduce $\mathrm{Ga}$ contamination.

APT investigations were done at KAUST (Thuwal, Saudi Arabia) using a LAWATAP (laser assisted wide-angle tomographic atom probe) and a LEAP 4000X HR (local electrode atom probe) system (CAMECA Instruments). Samples were measured at $35 \mathrm{~K}$ : at the LAWATAP with a pulse rate of $10 \mathrm{kHz}$ and a flux rate between 0.01 and 0.05 atoms/pulse, at the LEAP with a pulse rate of $100 \mathrm{kHz}$ and a pulse fraction of $15 \%$. Energy resolution is better at LEAP 4000X HR than at LAWATAP, while detection efficiency is lower $(\sim 0.37$ vs $\sim 0.60)$.

To set up a state of thermal equilibrium, electricalresistivity measurements were done for a heating and cooling run (Fig. 1). With a waiting time of $24 \mathrm{~h}$ for any aging step (data were taken at liquid-nitrogen temperature), thermal equilibrium could only be reached down to $823 \mathrm{~K}$ in the cooling run. Prolonged aging for 16 days was sufficient to set up thermal equilibrium at $723 \mathrm{~K}$. At $673 \mathrm{~K}$ no thermal equilibrium was reached within 119 days.

For the diffuse scattering experiment, a slice with a $\langle 210\rangle$ surface normal was cut by spark erosion, being $2.5 \mathrm{~mm}$ in thickness and $9 \mathrm{~mm}$ in diameter. The sample was homogenized for $48 \mathrm{~h}$ at 1123 to $1173 \mathrm{~K}$ under Ar gas, 
TABLE I. Pairs $(i j)$ with largest scattering contrast $\Delta_{i j}=\mid f_{i}-$ $\left.f_{j}\right|^{2}$ at $20 \mathrm{eV}$ below the absorption edges of $\mathrm{Cr}, \mathrm{Fe}$, and $\mathrm{Ni}$.

\begin{tabular}{lccc}
\hline \hline Energy $(\mathrm{eV})$ & \multicolumn{3}{c}{ Pair $(i j)$ and $\Delta_{i j}(\mathrm{eu})$} \\
\hline 5969 & Cr-Ni 70.5 & Cr-Co 51.5 & Cr-Fe 34.4 \\
7092 & Fe-Ni 30.1 & Fe-Co 19.3 & Fe-Cr 14.5 \\
8313 & Ni-Co 17.8 & Ni-Fe 14.9 & Ni-Cr 7.0 \\
\hline \hline
\end{tabular}

and sand quenched. Aging was done for 41 days at $723 \mathrm{~K}$, followed by water quench. A breaking of glass was avoided as samples were prone to heat blushing. The slice was polished and finally shortly treated electrochemically using methanol with hydrochloric acid (10\%). The latter step was required as to eliminate any indication of Debye-Scherrer rings in scattering experiments.

Diffuse x-ray scattering was done at RT on the ID01 beamline (ESRF, Grenoble). The diffractometer was additionally equipped with a $\chi$ and a $\phi$ circle for approximating a standard four-circle goniometer, but with a restricted $\chi$ range of $0^{\circ}$ to $28^{\circ}$. With a $\langle 210\rangle$ sample surface, this allowed us to address the 020 and 220 Bragg reflections for sample orientation and to investigate the scattering at $\left(h_{x}, h_{y}, 0\right)$ positions, using the symmetrical Bragg-Brentano configuration. A Vitus H80 silicon drift detector (KETEK GmbH) and a Vortex-EM silicon drift detector (Hitachi), both with an energy resolution of about $270 \mathrm{eV}$ FWHM, were employed. Data were taken under time control, employing RRS/fluorescence lines for monitoring the variation in beam intensity.

Scattering was taken at $20 \mathrm{eV}$ below the $K$ absorption edges of $\mathrm{Cr}$ (at $5969 \mathrm{eV}$ ), $\mathrm{Fe}$ (at $7092 \mathrm{eV}$ ), and $\mathrm{Ni}$ (at $8313 \mathrm{eV}$ ). These three cases are subsequently also referred to as "Cr edge", "Fe edge", and "Ni edge". The uncertainty in energy of the incoming photons was $1 \mathrm{eV}$. This choice in energy allowed sufficient variation in Hönl corrections while keeping uncertainties in $K-M_{\mathrm{II}} M_{\mathrm{III}}$ RRS small (cf. Ref. [1]). For these three measurements, the largest values for scattering contrast $\Delta_{i j}=\left|f_{i}-f_{j}\right|^{2}, f_{i}=$ atomic scattering factor of component $i$ (data taken from Ref. [36]) are given in Table I. Here values are presented for scattering vector $\mathbf{h}=0$, Hönl corrections were taken from Ref. [37]. The penetration depth of the $\mathrm{x}$ rays, the inverse of the linear absorption coefficient, ranges between $14 \mu \mathrm{m}$ at the Cr edge and $6 \mu \mathrm{m}$ at the Ni edge.

Energy-resolved spectra were registered at $\left(h_{x}, h_{y}, 0\right)$ positions with $h_{x} \leqslant h_{y}$ on a fixed grid of $0.1 \mathrm{rlu}$. Data were taken at 450 to 680 positions up to $110^{\circ}$ in scattering angle. The counting time per position was $30 \mathrm{~s}$. Typical values in diffuse scattering (comprising mainly the line of elastic scattering and thermal diffuse scattering) were 6000 counts near the $\mathrm{Cr}$ and Fe edge and 11000 near the Ni edge.

Due to the closeness in energy between elastic scattering and the various inelastic contributions (Compton scattering, fluorescence with noticeable escape peaks, RRS $K-L_{\text {II }} L_{\text {III }}$, RRS $K-M_{\mathrm{II}} M_{\mathrm{III}}$, and thermal diffuse scattering, together with energy spread by the detection process), the following strategy was chosen to obtain elastic scattering from the energyresolved spectra.

(1) The intensity ratio $\mathrm{Cr} K_{\beta} / \mathrm{Cr} K_{\alpha}$ was determined to $0.20 \pm 0.005$, this value was also used to obtain $\mathrm{Cr} K-M_{\mathrm{II}} M_{\mathrm{III}}$ from $\mathrm{Cr} K-L_{\text {II }} L_{\text {III }}$. The same value was employed to determine Fe $K-M_{\mathrm{II}} M_{\mathrm{III}}$ and Ni $K-M_{\mathrm{II}} M_{\mathrm{III}}$ from the respective $K-L_{\mathrm{II}} L_{\mathrm{III}}$ RRS.

(2) Compton scattering was considered to be negligible by taking only the higher-energy part of the elastic scattering region, which was then multiplied by 2 (plus adding the maximum in scattering) to obtain elastic scattering plus TDS.

(3) Thermal diffuse scattering was calculated up to third order using the experimental values of the elastic constants $c_{i j}$ and a "gray" scattering factor squared of $\left|\sum_{i=1,4} c_{i} f_{i}\right|^{2}$. The elastic constants $c_{i j}$ for $\mathrm{Cr}$-Fe-Co-Ni were determined by the pulse-echo-overlap method using a cylinder with a $\langle 110\rangle$ axis prepared from the same single crystal. They were $c_{11}=217(2) \mathrm{GPa}, c_{12}=138(2) \mathrm{GPa}, c_{44}=133(2) \mathrm{GPa}$. On the basis of these data, a thermal Debye-Waller factor $\exp \left[-2 B_{\mathrm{th}}(\sin \theta / \lambda)^{2}\right](\theta=$ half the scattering angle, $\lambda$ $=$ wavelength of incoming photons) with $B_{\mathrm{th}}=0.316 \times$ $10^{-2} \mathrm{~nm}^{2}$ was determined.

(4) Calibration to Laue units was done by choosing a value such that the scattering within a small range around the 020 and 220 Bragg reflections (a radius of 0.35 rlu was taken) corresponds to thermal diffuse scattering. A contribution by SRO scattering (taken from electronic structure calculations) was also considered in calibrating data when SRO scattering was close to monotonic Laue scattering.

\section{ELECTRONIC STRUCTURE CALCULATIONS}

\section{A. Green's function calculations}

The electronic structure and the total energy of random $\mathrm{Cr}_{25} \mathrm{Fe}_{25} \mathrm{Co}_{25} \mathrm{Ni}_{25}$ alloys were calculated by the Green's function exact muffin-tin orbital (EMTO) [38,39] and projector augmented wave (PAW) [40,41] methods. In the Green's function EMTO calculations, substitutional disorder was treated either by using the coherent potential approximation (CPA) [42] as implemented in the EMTO-CPA method [43] or/and by using the locally self-consistent Green's function (LSGF) technique [44] within the EMTO method, ELSGF [45]. The ELSGF method accurately accounts for the local environment effects in random alloys and has been used here (i) for tuning and testing the EMTO-CPA method, which was mostly used in calculations of magnetic and chemical interactions, and (ii) for calculating room temperature elastic constants of random $\mathrm{Cr}_{25} \mathrm{Fe}_{25} \mathrm{Co}_{25} \mathrm{Ni}_{25}$ with and without atomic SRO.

All EMTO-CPA calculations were done by the Lyngby version of the Green's function EMTO code [46], where the screened Coulomb interactions in the single-site DFTCPA approximation [47] and screened generalized perturbation method (SGPM) $[31,48,49]$ are implemented. The selfconsistent electronic structure calculations were done in the local density approximation (LDA) using the Perdew and Wang functional [50], while the total energies were calculated by the full charge-density technique [39] in the PerdewBurke-Ernzehof generalized gradient approximation (PBEGGA) [51]. The Brillouin zone integrations were done using a $28 \times 28 \times 28$ Monkhorst-Pack grid [52] for the fcc structure and a $28 \times 28 \times 18$ grid for the hcp structure. All the calculations have been done with $l_{\max }=3$ for partial waves and the electronic core states were recalculated at every iteration during the self-consistent calculations for valence electrons. 
The ELSGF method was also used to calculate the screened Coulomb interactions used in the DFT-CPA part of the EMTO-CPA calculations. In this case, the one-electron potential of the alloy components and the total energy have additional contributions, $V_{\mathrm{scr}}^{i}$, and $E_{\mathrm{scr}}$, respectively, due to the screening charge around atomic spheres, which is not accounted for in the single-site approximation [47]

$$
\begin{aligned}
& V_{\mathrm{scr}}^{i}=-e^{2} \alpha_{i}^{0} \frac{\bar{q}_{i}}{S_{\mathrm{WS}}}, \\
& E_{\mathrm{scr}}=\sum_{i} c_{i} E_{\mathrm{scr}}^{i}, \quad E_{\mathrm{scr}}^{i}=-e^{2} \frac{1}{2} \alpha_{i}^{0} \beta_{\mathrm{scr}} \frac{\bar{q}_{i}^{2}}{S_{\mathrm{WS}}} .
\end{aligned}
$$

Here $\bar{q}_{i}$ and $\alpha_{i}^{0}$ are the net charge of the atomic sphere of the $i$ th component in the single-site CPA calculations and its on-site screening constant, respectively; $S_{\mathrm{WS}}$ is the WignerSeitz radius; $E_{\mathrm{scr}}^{i}$ is the contribution of the screened Coulomb interactions to the electrostatic energy of the $i$ th alloy component; and $\beta_{\text {scr }}$ is the average on-site screening constant, which accounts for the electrostatic multipole moment energy contribution due to the inhomogeneous local environment of different sites in a random alloy.

The screening constants were determined from ELSGF 864-atom and 512-atom supercell calculations for fcc and hcp $\mathrm{Cr}-\mathrm{Fe}-\mathrm{Co}-\mathrm{Ni}$ alloys. The on-site screening constants $\alpha_{i}^{0}$ were determined from the conditional average of the net charges $q_{i}$ and the Madelung potentials $V_{i}^{\text {Mad }}$ of the $i$ th component in the supercell, $\left\langle q_{i}\right\rangle$ and $\left\langle V_{i}^{\mathrm{Mad}}\right\rangle$, respectively, as

$$
\alpha_{i}^{0}=\frac{S_{\mathrm{WS}}\left\langle V_{i}^{\mathrm{Mad}}\right\rangle}{e^{2}\left\langle q_{i}\right\rangle} .
$$

The intersite screening constants $\alpha_{p}^{i j}$ (hint: not to be confused with the partial SRO parameter $\alpha_{l m n}^{i j}$ ) are needed in the calculations of the electrostatic contribution $V_{p}^{i j-\mathrm{scr}}$ to the SGPM potential at the $p$ th coordination shell for the $i-j$ pair of alloy components

$$
V_{p}^{i j-\mathrm{scr}}=e^{2} \alpha_{p}^{i j} \frac{\bar{q}_{i j}^{2}}{S_{\mathrm{WS}}} .
$$

Here $\bar{q}_{i j}=\bar{q}_{i}-\bar{q}_{j}$ were obtained in the supercell ELSGF calculations for random alloys from the screening charge by exchanging the corresponding alloy components $i$ and $j$ (each of them having random local environment on average), as described in Ref. [47].

\section{B. Projector augmented wave calculations}

The projector augmented wave (PAW) method [40,41] was used as implemented in the Vienna ab initio simulation package (VASP) code [53-55] to calculate the total energy and local lattice relaxations in fcc and hcp random $\mathrm{Cr}_{25} \mathrm{Fe}_{25} \mathrm{Co}_{25} \mathrm{Ni}_{25}$ alloys using $4 \times 4 \times 4(\times 4)$ (256-atom) supercells for the fcc structure and $6 \times 6 \times 4(\times 2)(288$-atom) and $8 \times 4 \times 4(\times 2)(256$-atom) supercells for the hcp structure. In all the cases, the SRO parameters at the first seven coordination shells were minimized for all the pairs of alloy components to get an atomic distribution close to a random one. In particular, the SRO parameters were equal to zero at the first two (for fcc) and three (for hcp) coordination shells and less than 0.03 in magnitude at the next four coordination shells. The PBE generalized gradient approximation was used in self-consistent PAW calculations [51]. The Brillouin zone integrations were done using a $4 \times 4 \times 4$ MonkhorstPack grid [52] for the fcc supercells (test calculations with a $6 \times 6 \times 6$ grid changed the total energy only by about $1 \mu \mathrm{eV} /$ atom) and $4 \times 4 \times 3$ for the 288 -atom hcp supercell and $2 \times 4 \times 3$ for the 256 -atom hcp supercell. The energy cutoff was $400 \mathrm{eV}$.

\section{RESULTS FROM APT}

The composition of the material was determined by LEAP investigations, using six tips. Doubly charged ions were considered in analyzing the mass spectra. Time-of-flight overlap is only noted for ${ }^{54} \mathrm{Cr}$ and ${ }^{54} \mathrm{Fe}$ (with low $\mathrm{Cr}$ and $\mathrm{Fe}$ isotope abundances), ${ }^{58} \mathrm{Ni}$ and ${ }^{58} \mathrm{Fe}$ (with high $\mathrm{Ni}$, low $\mathrm{Fe}$ isotope abundance). With 23.8 at.\% Cr, 25.7 at.\% Fe, 25.1 at.\% Co, 25.4 at. $\% \mathrm{Ni}$, the constituents are close to their starting atomic fraction. The largest difference is seen with $\mathrm{Cr}$. A reason for this might be that $\mathrm{Cr}$ has the lowest field evaporation strength among the four elements (Cr: $29 \mathrm{~V} / \mathrm{nm}$; Co: $32 \mathrm{~V} / \mathrm{nm}$; Fe: $33 \mathrm{~V} / \mathrm{nm}$; Ni: $34 \mathrm{~V} / \mathrm{nm}$; values for the elements at $0 \mathrm{~K}$ and doubly charged ions) [11]. Thus, $\mathrm{Cr}$ is preferentially subject to field evaporation between the voltage pulses, i.e., such an ion is then attributed to the background.

The reconstructed volume of the as-grown material (LEAP investigation) shows no signs of order or decomposition [Fig. 2(a) illustrates the $\mathrm{Cr}$ atoms, the same feature is seen with $\mathrm{Fe}, \mathrm{Co}, \mathrm{Ni}$ atoms]. For a closer investigation of site occupation (LAWATAP investigation), the atom vicinity algorithm $[12,56]$ was applied using $\mathrm{Ni}$ atoms in the neighborhood of the 002 pole as a reference atom. As vicinity a cube with an edge length of $1 \mathrm{~nm}$ was chosen. The cross correlation to the density profiles of the four components along [001] is shown in Fig. 2(b). One notices that the planes perpendicular to [001] (they show up by a positive value in the cross correlations) do not differ in magnitude. Thus, the four fcc sublattices are equally occupied by the four elements. The slight differences in the onset of a neighbored-plane range between the elements follows in the sequence $\mathrm{Cr}, \mathrm{Ni}, \mathrm{Co}, \mathrm{Fe}$. If one assumes that a fcc lattice with random site occupation is present, one would expect a sequence as given by the field evaporation strengths of the pure elements [12,57], i.e., the sequence $\mathrm{Cr}, \mathrm{Co}, \mathrm{Fe}$, $\mathrm{Ni}$. The different finding indicates that local order might be present in the alloy. As ions with the lowest field evaporation strength are reconstructed closer to the center of a pole, there is a slight increase in the $\mathrm{Cr}$ fraction along the 002 pole [Fig. 2(c)]. The apparently enhanced $\mathrm{Cr}$ fraction may not be taken as a sign of local decomposition. It is, however, a good indicator for the [001] axis and was also found for aged samples.

\section{RESULTS FROM SCATTERING}

\section{A. Energy spectra}

Figure 3 shows parts of the energy-resolved spectra close to elastic scattering. They provide averaged views of the asmeasured spectra at the three edges, as they were obtained by 
(a)

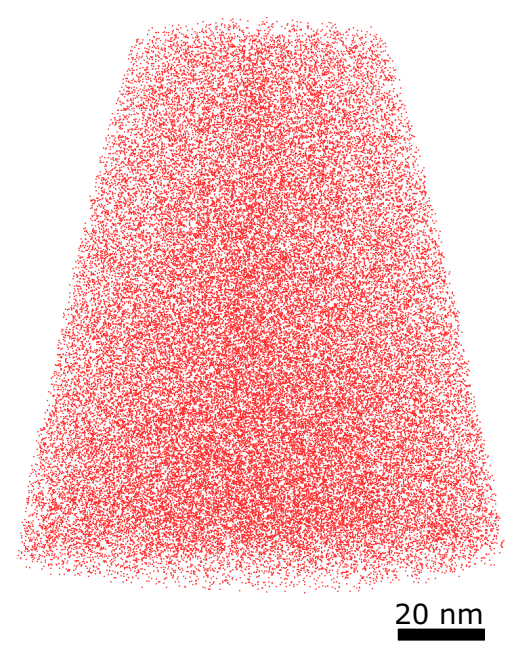

(b)

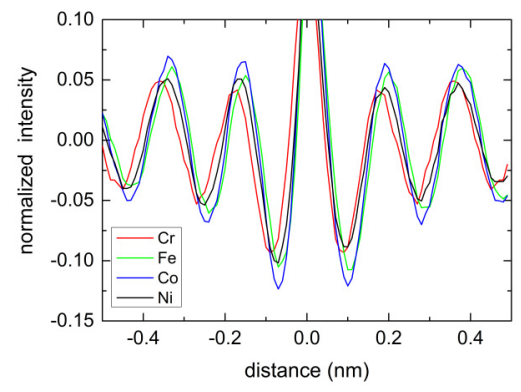

(c)

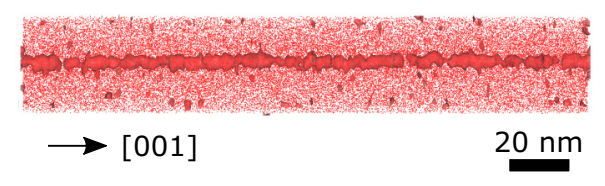

FIG. 2. (a) Reconstruction of the evaporated volume from Cr-FeCo-Ni (as grown). Only $\mathrm{Cr}$ atoms are marked. (b) Cross-correlated density profiles with $\mathrm{Ni}$ as reference atom and $\mathrm{Cr}, \mathrm{Fe}, \mathrm{Co}, \mathrm{Ni}$ at the 002 pole. An increase in the field evaporation strength $\mathrm{Cr} \rightarrow$ $\mathrm{Ni} \rightarrow \mathrm{Co} \rightarrow \mathrm{Fe}$ is noted. (c) This evaporation sequence results in an apparent $\mathrm{Cr}$ fraction increase along [001] as revealed by the red isosurface of 26.5 at.\% $\mathrm{Cr}$ that confines the high- $\mathrm{Cr}$ region around the 002 pole.

adding up the spectra for all $\mathbf{h}$ positions (shown in black). In addition, the separated $K-L_{\mathrm{II}} L_{\mathrm{III}}$ lines are shown in red. The onset of the respective $K-M_{\mathrm{II}} M_{\mathrm{III}} \mathrm{RRS}$ is shifted by $-42 \mathrm{eV}$ (Cr edge), $-53 \mathrm{eV}$ (Fe edge), $-67 \mathrm{eV}$ (Ni edge) off the energy of the incoming photons. As the $K-M_{\mathrm{II}} M_{\mathrm{III}} \mathrm{RRS}$ is lower in intensity than $K-L_{\mathrm{II}} L_{\mathrm{III}} \mathrm{RRS}$ by a factor of $\sim 5.0$, it could not be separated directly in the energy spectra, but was determined by scaling the $K-L_{\mathrm{II}} L_{\mathrm{III}} \mathrm{RRS}$. If one compares the $K-M_{\mathrm{II}} M_{\mathrm{III}}$ RRS with respect to elastic scattering plus TDS, there is a clear tendency: The ratio between them increases from 0.06 (Cr edge) over 0.09 (Fe edge) to 0.24 (Ni edge). This increase reflects the concurrent decrease in the scattering contrast [cf. Table I and Eq. (3)].

At the Cr edge [Fig. 3(a)] the lower peak at ch. 2685 is the $\mathrm{Cr} K-L_{\mathrm{II}} L_{\mathrm{III}} \mathrm{RRS}$, shifted by the binding energies (a)

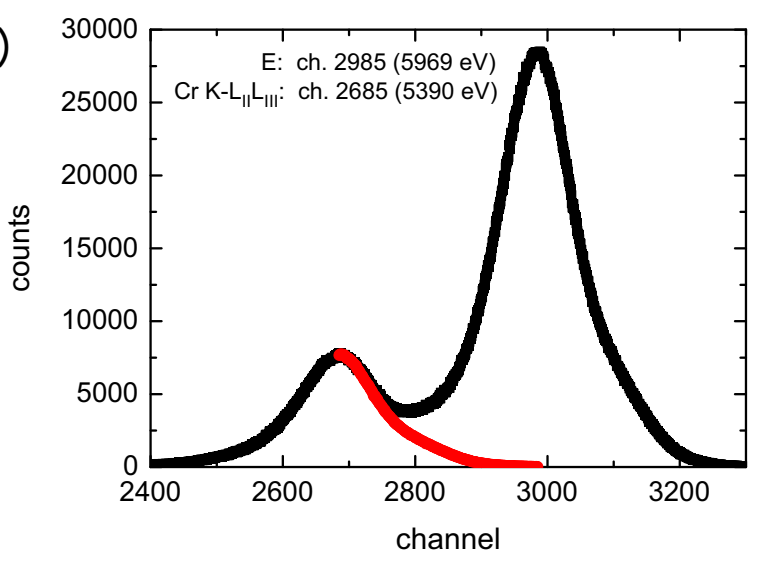

(b)

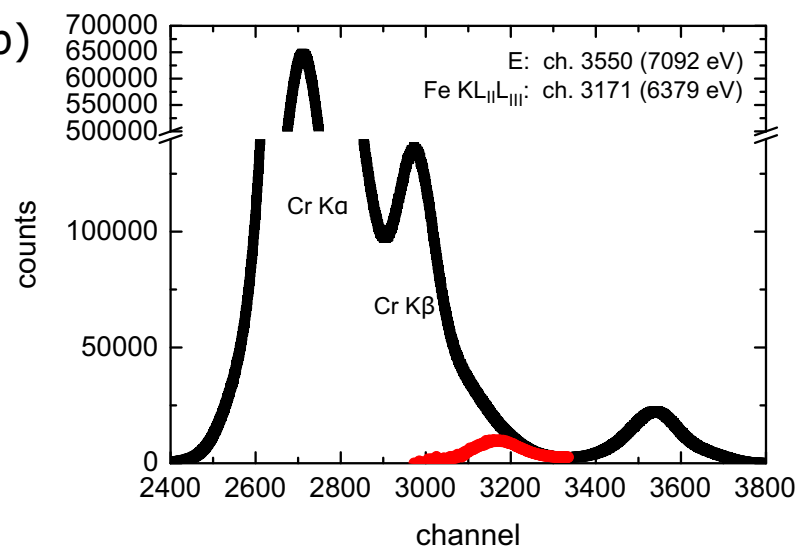

(c)

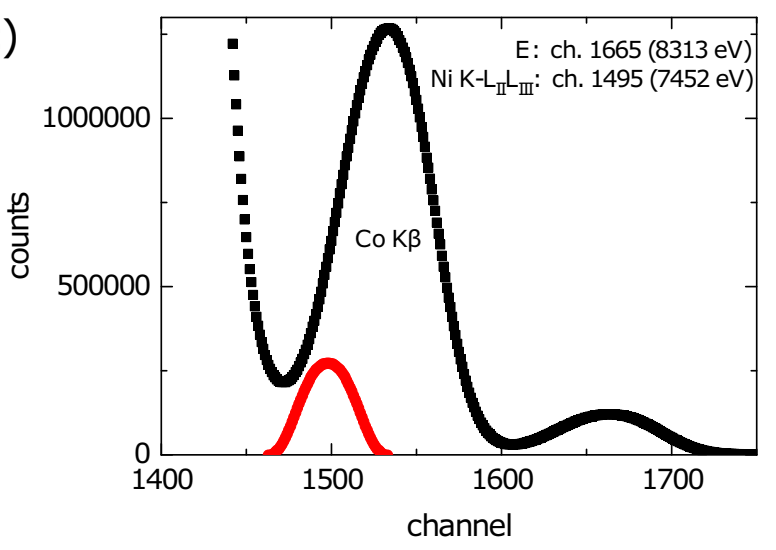

FIG. 3. Energy-resolved spectra (a) at the $\mathrm{Cr}$ edge, (b) Fe edge (scale: $1 \mathrm{ch} . \approx 2.0 \mathrm{eV}$ ), and (c) Ni edge $(1 \mathrm{ch} . \approx 5.0 \mathrm{eV})$ around the lines that contain elastic scattering of energy $E$. To provide an average view, the spectra at all $\mathbf{h}$ positions (in black) were added. The $K-L_{\mathrm{II}} L_{\mathrm{III}}$ resonant Raman scattering is shown in red. Channel numbers refer to the maximum position of the respective lines, with theoretical energy values in parentheses.

$L_{\mathrm{II}}, L_{\mathrm{III}}$ from elastic scattering. The shape of the $\mathrm{Cr} K-L_{\mathrm{II}} L_{\mathrm{III}}$ RRS shows no indication for the high-energy threshold: The smearing in energy due to the resolution of the silicon drift detector dominates. At the Fe edge and the Ni edge [Figs. 3(b) 
and 3(c)] the slight bumps at ch. 3171 and ch. 1495, respectively, are the $K-L_{\mathrm{II}} L_{\mathrm{III}}$ RRS.

With increasing scattering angle, Compton scattering gets shifted towards lower energies: For the maximum scattering angle of $110^{\circ}$ in the present investigation, the shift is $94 \mathrm{eV}$ (Cr edge), $132 \mathrm{eV}$ (Fe edge), $181 \mathrm{eV}$ (Ni edge). Also, total Compton scattering gets increasingly smeared according to the momentum distribution of the valence electrons. On the basis of the tabulated values by Biggs et al. [58] (see also Ref. [59] for the nomenclature), the FWHM of this spread in Compton scattering was determined to about $168 \mathrm{eV}(\mathrm{Cr}$ edge), $198 \mathrm{eV}$ (Fe edge), $231 \mathrm{eV}$ (Ni edge) at the largest scattering angle. Finally, this smeared profile is still convoluted with the energy resolution of the detectors (FWHM is $\sim 270 \mathrm{eV}$ ). As a consequence, only the lower energy half of the $\mathrm{Cr} K-L_{\text {II }} L_{\text {III }}$ RRS (Cr edge) and of the Co $K_{\beta}$ fluorescence line ( $\mathrm{Ni}$ edge) were employed for monitoring the incoming beam intensity.

\section{B. Elastic diffuse scattering}

The elastic diffuse scattering in Laue units is shown in Fig. 4 (upper triangles). At the $\mathrm{Cr}$ edge and the $\mathrm{Fe}$ edge [Figs. 4(a) and 4(b)], one notices diffuse maxima close to $X$ position. With 2.8 to $3.5 \mathrm{Lu}$, the maxima are higher at the $\mathrm{Cr}$ edge than at the $\mathrm{Fe}$ edge where they amount to 1.8 to 2.5 Lu. No indication for an increase in scattering towards the Bragg reflections is seen outside a distance of $0.35 \mathrm{rlu}$. For $|\mathbf{h}|<0.6$ rlu there is a slight increase in scattering that might indicate a slight tendency to local decomposition, in addition to the dominant sign of local order. Whether this increase at small $\mathbf{h}$ positions is due to background not properly considered is unknown. The neglect of Compton scattering in the present evaluation, however, is barely the reason: (i) Total Compton scattering only amounts to $5 \%$ to $30 \%$ of the elastic scattering at scattering vectors below 0.6 rlu. (ii) Total Compton scattering increases with increasing scattering vector. Its tentative consideration in data evaluation would thus lead to an enhanced increase in scattering with increasing scattering vector.

Indications for displacement scattering are seen at the $\mathrm{Cr}$ edge and the $\mathrm{Fe}$ edge [Figs. 4(a) and 4(b)]: The diffuse maxima are not exactly located at $X$ position but shifted systematically, as seen best at the $(1,1,0)$ and the $(1,2,0)$ position. Also, with increasing distance from the $(0,2,0)$ position, elastic diffuse scattering increases faster along $[0,1,0]$ than along $[0,-1,0]$.

At the Ni edge [Fig. 4(c)] the elastic diffuse scattering shows very small modulations, due to the low scattering contrast (see Table I). The scattering pattern appears much rougher than at the other two edges: The counting statistics of about $1 \%$ for the registered scattering already leads to an uncertainty of $5 \%$ in the elastic scattering, as $\mathrm{Ni} K-M_{\mathrm{II}} M_{\mathrm{III}}$ RRS and the calculated TDS comprise $80 \%$ of the registered scattering.

\section{Short-range order and static atomic displacements}

Elastic diffuse scattering shown in Fig. 4 (upper triangles) was analyzed under the assumption that Huang scattering is (a)

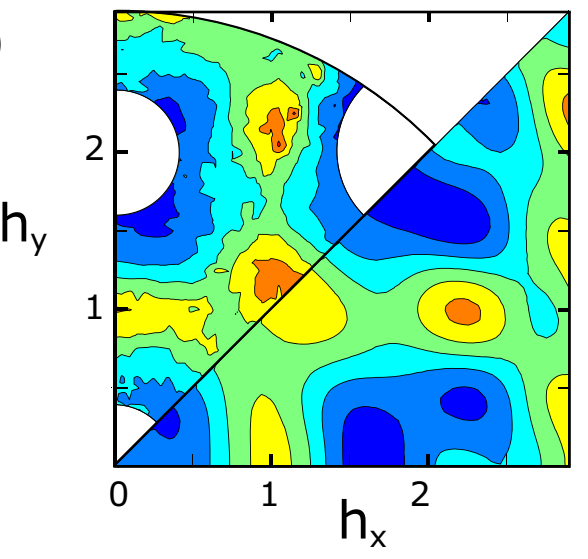

(b)

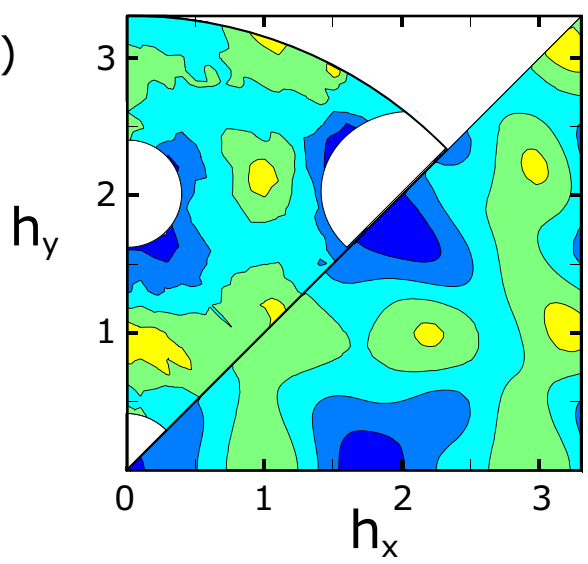

(c)

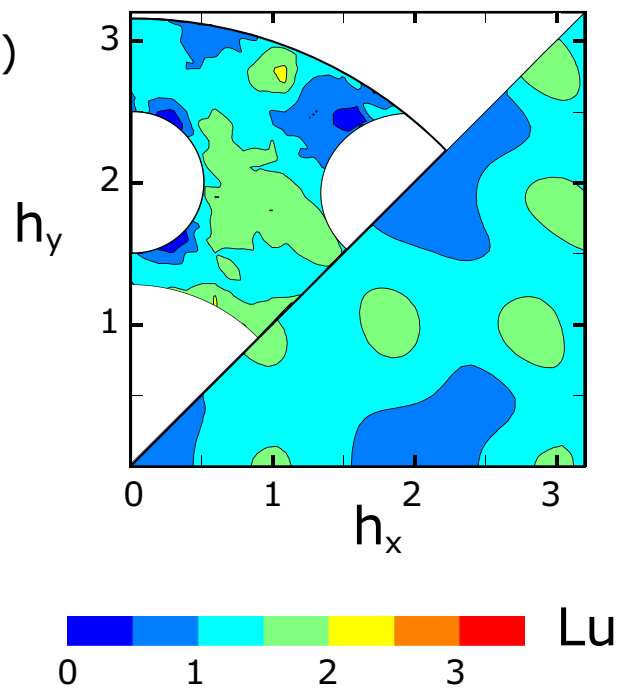

FIG. 4. Elastic diffuse scattering at $\left(h_{x}, h_{y}, 0\right)$ positions in Laue units as measured (upper triangles, $h_{x}<h_{y}$ ) and as fitted (lower triangles, $h_{x}>h_{y}$ ): (a) at the Cr edge, (b) at the Fe edge, and (c) at the Ni edge.

negligible. The resulting effective SRO and SE parameters $\alpha_{l m n}^{\text {eff }}$ and $\gamma_{l m n}^{x \text {,eff }}$ from least-squares fitting are given in Table II. Only a restricted number of parameters is determinable (at most five $\alpha_{l m n}^{\text {eff }}$ and seven $\gamma_{l m n}^{x, \text { eff }}$ ) as no 3D data sets were taken. The unweighted $R$ values of the fits were 0.13 at the Cr edge, 0.15 at the $\mathrm{Fe}$ edge, and 0.24 at the Ni edge. Table II reveals the following general features: (i) At all three edges, $\alpha_{000}^{\text {eff }}$ 
TABLE II. Effective Warren-Cowley short-range order parameters $\alpha_{l m n}^{\text {eff }}$ and effective linear displacement parameters $\gamma_{l m n}^{x, \text { eff }}$ of $\mathrm{Cr}-\mathrm{Fe}-\mathrm{Co}-\mathrm{Ni}$ after aging at $723 \mathrm{~K}$, as determined at the $\mathrm{Cr}$ edge, the $\mathrm{Fe}$ edge, and the $\mathrm{Ni}$ edge from diffuse scattering. Also given are $\alpha_{l m n}^{\text {eff }}$ from electronic structure calculations, employing least-squares fitting to calculated data exclusively at $\left(h_{x}, h_{y}, 0\right)$ positions (cf. Sec. VII H).

\begin{tabular}{lccc}
\hline \hline & & $\alpha_{l m n}^{\text {eff }}($ scattering $)$ & \\
\cline { 2 - 4 }$l m n$ & $5969 \mathrm{eV}$ & $7092 \mathrm{eV}$ & $8313 \mathrm{eV}$ \\
\hline 000 & $1.136(25)$ & $1.093(24)$ & $1.142(30)$ \\
110 & $-0.125(3)$ & $-0.089(5)$ & $-0.044(5)$ \\
200 & $0.097(11)$ & $0.073(10)$ & $0.034(11)$ \\
211 & $0.022(2)$ & $0.000(3)$ & $-0.004(3)$ \\
220 & $0.015(5)$ & & \\
& & $\gamma_{l m n}^{x}$ eff $($ scattering & \\
110 & $0.016(2)$ & $0.016(2)$ & $-0.010(3)$ \\
200 & $-0.062(6)$ & $-0.035(8)$ & $0.001(11)$ \\
121 & $-0.009(1)$ & $-0.004(2)$ & $0.006(2)$ \\
211 & $0.002(1)$ & $0.004(1)$ & $0.005(2)$ \\
220 & $0.037(3)$ & $0.023(4)$ & $-0.007(5)$ \\
& & $\alpha_{l m n}^{\text {eff }}($ theory $)$ & \\
000 & $1.0457(101)$ & $1.0113(37)$ & $0.9951(5)$ \\
110 & $-0.1294(12)$ & $-0.0518(4)$ & $0.0056(1)$ \\
200 & $0.1696(46)$ & $0.0690(17)$ & $0.0112(2)$ \\
211 & $0.0126(6)$ & $0.0050(2)$ & $0.0029(1)$ \\
220 & $0.0312(20)$ & $0.0090(7)$ & $-0.0060(1)$ \\
310 & $-0.0472(8)$ & $-0.0175(3)$ & $-0.0010(1)$ \\
\hline \hline
\end{tabular}

is close to 1 , the theoretical value for any $\alpha_{000}^{i j}$. As $\alpha_{110}^{\text {eff }}$ is negative, local order always prevails. The signs of the $\alpha_{l m n}^{\text {eff }}$ agree with those of the $L 1_{2}$ structure except for $l m n=211$, a feature repeatedly noted for binary alloys with a low degree of the $L 1_{2}$ type of short-range order (see, e.g., the compilation of Ni-rich Ni-Al, Table 3 in Ref. [1]). (ii) Both the apparent degree of local order as expressed by $\alpha_{l m n}^{\text {eff }}$ and the apparent strength of the $\gamma_{l m n}^{x, \text { eff }}$, decrease with decreasing strength of the scattering contrast.

Using the fitted parameters of Table II, elastic diffuse scattering was recalculated for $\left(h_{x}, h_{y}, 0\right)$ positions at the three edges (Fig. 4, lower triangles). The scattering patterns confirm the location of the diffuse maxima close to the $X$ position for the $\mathrm{Cr}$ edge and the $\mathrm{Fe}$ edge. Their values in the recalculated scattering are slightly lower than those of the measured diffuse maxima: $2.5 \mathrm{Lu}$ at the $\mathrm{Cr}$ edge and $2.0 \mathrm{Lu}$ at the Fe edge. This underestimate might be due to the restricted number of fitted parameters. SRO scattering (Fig. 5) shows a platelike pattern around its maximum at $X$ positions also frequently seen with the $L 1_{2}$ type of short-range order (see the textbook example in Warren [60]). At the Ni edge where no maxima could be resolved in Fig. 4(c), a maximum of 1.7 $\mathrm{Lu}$ is now seen close to $X$ position.

\section{FIRST-PRINCIPLES RESULTS}

\section{A. Energetics of random $\mathrm{Cr}_{25} \mathrm{Fe}_{25} \mathrm{Co}_{25} \mathrm{Ni}_{25}$ at 0 K: ELSGF vs PAW}

The electronic structure and total energy of random $\mathrm{Cr}_{25} \mathrm{Fe}_{25} \mathrm{Co}_{25} \mathrm{Ni}_{25}$ were calculated in the fcc and hcp
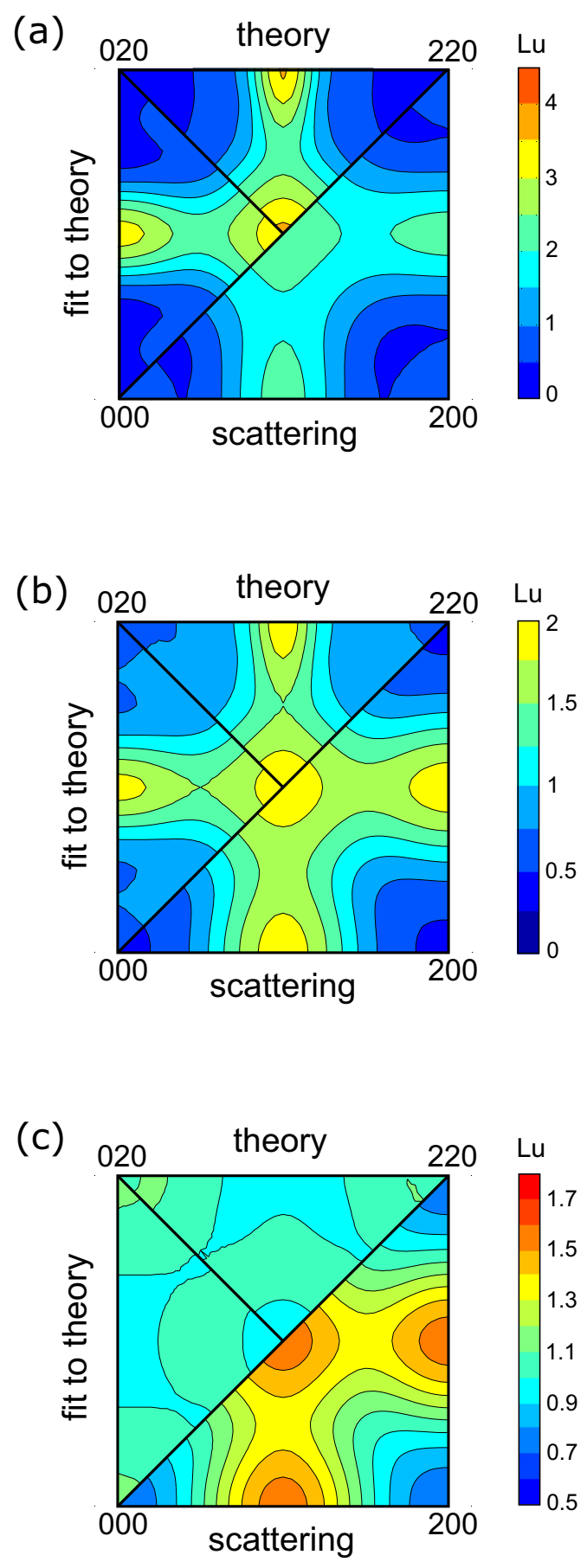

FIG. 5. SRO scattering at $\left(h_{x}, h_{y}, 0\right)$ positions as determined from diffuse scattering based on Table II (lower triangles) and from the present electronic structure calculations at $720 \mathrm{~K}$ [(top sectors) and (left sectors) based on Table II]: (a) at the $\mathrm{Cr}$ edge, (b) at the $\mathrm{Fe}$ edge, and (c) at the Ni edge.

structures by the ELSGF method using 864-atom and 512atom supercells, respectively. In both cases, the local interaction zone comprised 18 nearest neighbors (the first two coordination shells in the fcc structure and three coordination shells in the hcp structure) together with the central atom. The atomic SRO parameters of both supercells were minimized for all pairs of alloy components up to the seventh coordination 


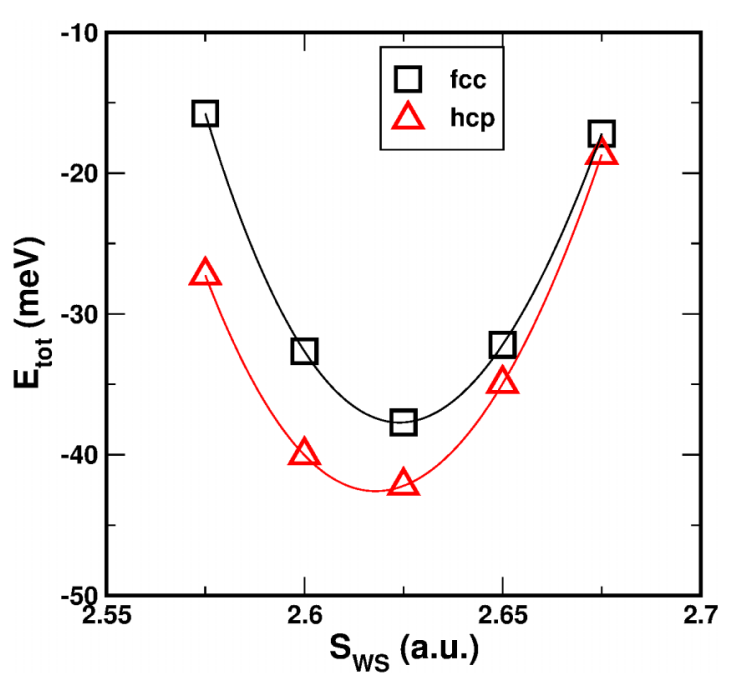

FIG. 6. Total energies of random fcc and hcp $\mathrm{Cr}_{25} \mathrm{Fe}_{25} \mathrm{Co}_{25} \mathrm{Ni}_{25}$ alloys from ELSGF calculations, as function of the Wigner-Seitz radius.

shell. The SRO parameters of the coordination shells included in the local interaction zone were equal to zero. For the hcp structure the ideal lattice-parameter ratio $c / a$ was used.

The fcc and hep total energies of random $\mathrm{Cr}_{25} \mathrm{Fe}_{25} \mathrm{Co}_{25} \mathrm{Ni}_{25}$ alloys calculated at $0 \mathrm{~K}$ are shown in Fig. 6 as function of the Wigner-Seitz radius. As one can see, the hcp structure has lower energy than the fcc one, or in other words, the fcc structure turns out to be unstable at $0 \mathrm{~K}$ in the present calculations.

Since the present version of ELSGF (as well as EMTO) cannot account for local lattice relaxations, and besides, the full charge density (FCD) calculations based on the EMTO self-consistent density within essentially atomic sphere approximation (ASA) lack real full-potential accuracy, the PAW method was used to check the fcc-hcp energy difference of $\mathrm{Cr}_{25} \mathrm{Fe}_{25} \mathrm{Co}_{25} \mathrm{Ni}_{25}$. A Wigner-Seitz radius of 2.625 a.u. was chosen, which is close to the theoretical PBE-GGA equilibrium value for the fcc structure at $0 \mathrm{~K}$.

The PAW calculations were done for both structures using two different supercells for every structure to get statistical averaging. The point is that supercell PAW calculations of supposedly random alloys suffer from the very approximate description of the electronic structure, which is incorrect (no Bloch states). Besides, the electronic structure and total energy in such calculations are quite sensitive to the underlying atomic configuration due to periodic boundary conditions in the electronic structure calculations. These boundary conditions effectively account for contributions from all atomic distribution correlation functions, including those which can never be optimized for a finite supercell. This is not the case of the LSGF method, which provides a correct electronic structure of random alloys without spurious contributions from long-distance atomic correlations and long-range order.

As in the case of the ELSGF calculations, the hcp structure is also more stable in the PAW calculations than the fcc one (by about $1.25 \mathrm{meV} /$ atom) if local atomic positions are unrelaxed. Allowing for local lattice relaxations, the energy difference changes very little and becomes about $1.43 \mathrm{meV} /$ atom, again in favor of the hcp structure. In fact, the energy of local lattice relaxation is about -6.58 and $-6.76 \mathrm{meV} /$ atom for the fcc and hcp structures, respectively, which is noticeably larger than the fcc-hcp energy difference, but obviously it is approximately the same for both structures.

Although the PAW calculations confirm the relative stability of the hcp structure of random $\mathrm{Cr}_{25} \mathrm{Fe}_{25} \mathrm{Co}_{25} \mathrm{Ni}_{25}$ at $S_{\mathrm{WS}}=$ 2.625 a.u., the ELSGF method yields a larger fcc-hcp energy difference of about $4.5 \mathrm{meV} /$ atom. Since the "exact" ELSGF (and EMTO in general) method is less accurate than PAW, the difference of about $3 \mathrm{meV} /$ atom between the PAW and ELSGF results can be attributed to some approximations in the ELSGF total energy calculations. Let us note that relative stability of the hcp structure in the FM ground state results in agreement with recent PAW calculations of the stacking fault energies (SFE) in this alloy $[61,62]$ where the SFE was found to be negative [63].

If one assumes that the error for the structural energy difference (of $3 \mathrm{meV} /$ atom) in the ELSGF calculations is constant in the range of $S_{\mathrm{WS}}$ shown in Fig. 6, the fcc structure becomes stable at $S_{\mathrm{WS}}$ close to the experimentally observed RT value, which is 2.644 a.u. (one notices in Fig. 6 that the energy difference between fcc and hcp structures decreases with increasing $S_{\mathrm{WS}}$ ). This was confirmed in the PAW supercell calculations of the fcc and hcp structures at $S_{\mathrm{WS}}=$ 2.644 a.u.: The fully relaxed fcc structure became more stable than the hcp structure by $0.7 \mathrm{meV} /$ atom. Since structural stability is not the topic of this work, it was assumed that these uncertainties have little effect on the effective magnetic and chemical interactions related to the corresponding, i.e., magnetic or chemical, configurational energetics on a fixed lattice.

\section{B. Electronic structure of random fec $\mathrm{Cr}_{25} \mathrm{Fe}_{25} \mathrm{Co}_{25} \mathrm{Ni}_{25}$ at $0 \mathrm{~K}$ : ELSGF vs EMTO-CPA}

The effective interaction calculations used for statistical modeling in this work were obtained in the CPA. To check the accuracy inherent in the CPA, the ELSGF method was used, which accounts for the local environment effects and provides the exact solution of the electrostatic problem within the approximations used for solving the Poisson equation and finding the electrostatic energy [47]. The main reason for possible problems is the quite different $d$-band filling of $\mathrm{Cr}$ and $\mathrm{Ni}$, which can substantially decrease the accuracy of the CPA due to substantial local environment effects. This was already reported for Ni-Cr binary alloys [24].

In Fig. 7 the electronic density of states (DOS) is shown as obtained for random fcc $\mathrm{Cr}_{25} \mathrm{Fe}_{25} \mathrm{Co}_{25} \mathrm{Ni}_{25}$ in the ELSGF supercell and EMTO-CPA calculations. Both calculations were done using exactly the same setup for the energy contour integration and $k$-points grid. The programs only differ in the treatment of electrostatics and local environment effects. The agreement is quite good (at least it is better than in the case of $\mathrm{Ni}-\mathrm{Cr}$ alloys [24]), especially close to the Fermi energy, which is important since the Fermi surface is responsible for many physical properties of alloys.

Although the size difference of the alloy components is relatively small, which results in a quite modest value of the local relaxation energy in random alloys (see above), the 


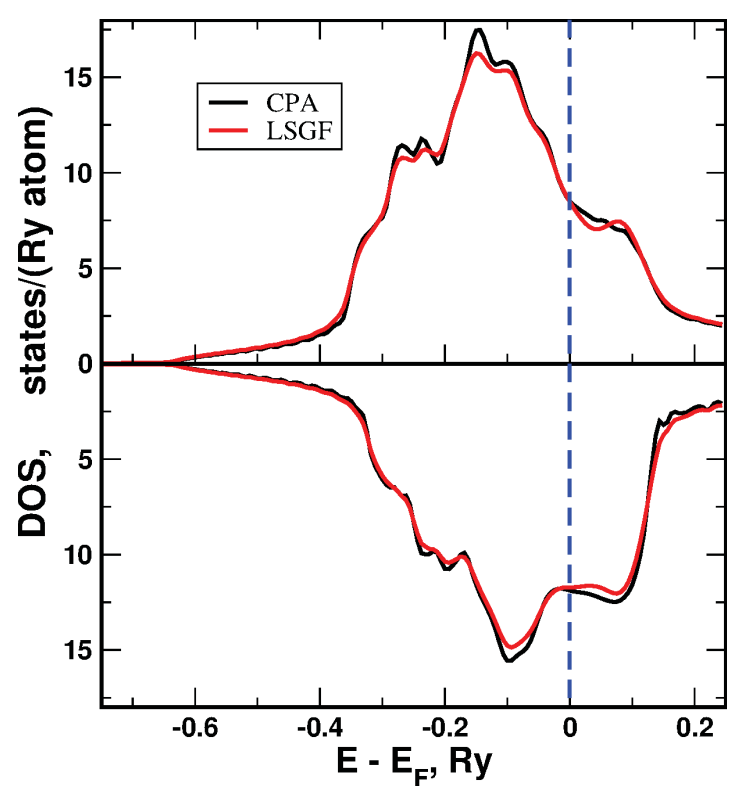

FIG. 7. Electronic density of states in random fcc $\mathrm{Cr}_{25} \mathrm{Fe}_{25} \mathrm{Co}_{25} \mathrm{Ni}_{25}$ from the ELSGF and EMTO-CPA calculations.

charge transfer effects are not negligible: The atomic spheres are gaining (or losing) about $0.1-0.2$ electron depending on the alloy component. Therefore, the correct description of electrostatics is important for the total energy and the effective interactions in the EMTO-CPA calculations. In Table III the results are given for the on-site screening constants $\alpha_{i}^{0}$ using Eq. (10), the conditional-average atomic sphere charges of alloy components in the ELSGF calculations, $\left\langle q_{i}\right\rangle$, and the atomic sphere charges in the EMTO-CPA calculations, $\bar{q}_{i}$, obtained with the corresponding on-site screening constants.

The agreement between $\left\langle q_{i}\right\rangle$ and $\bar{q}_{i}$ is very good, although there exists a quite substantial dispersion of individual local atomic charges of alloy components, which somehow follow a large scattering of local magnetic moments (which are not shown here since it is very similar to the one presented in Ref. [27]). The results for average magnetic moments are given in Table IV. It is obvious that the EMTO-CPA method is quite accurate in this case. Note that these are LDA results: The PBE-GGA spin magnetic moments are usually larger than those of LDA and experimental data for Fe.

\section{Modeling of paramagnetic state of $\mathrm{Cr}_{25} \mathrm{Fe}_{25} \mathrm{Co}_{25} \mathrm{Ni}_{25}$}

Random fcc $\mathrm{Cr}_{25} \mathrm{Fe}_{25} \mathrm{Co}_{25} \mathrm{Ni}_{25}$ is ferromagnetic (FM) at $0 \mathrm{~K}$, with some complex distribution of local magnetic mo- ments [27]. According to the experimental data [64,65], its Curie temperature is about $120-130 \mathrm{~K}$, which is quite low and thus, $\mathrm{Cr}_{25} \mathrm{Fe}_{25} \mathrm{Co}_{25} \mathrm{Ni}_{25}$ is in the paramagnetic state at ambient conditions. Since all the alloy components are itinerant magnets (which is reflected either in small magnitudes of local magnetic moments or their huge dispersion due to local environment effects-see Table IV and Ref. [27]), longitudinal spin fluctuations should play an important role in the paramagnetic state at finite temperature.

In this work it is assumed that the stiffness of transverse and longitudinal spin excitations are similar. Therefore, these two degrees of freedom are fully coupled for all alloy components (it is probably a less accurate approximation for $\mathrm{Fe}$ and Co, however, it is still a reasonable one in order to get a qualitative picture of magnetic and chemical interactions). In this case, neglecting magnetic SRO effects, the full magnetic entropy related to both transverse and longitudinal spin fluctuations (LSF) can be expressed as $[66,67]$

$$
S_{i}^{\text {magn }}=3 \ln \left(\langle m\rangle_{i}\right)
$$

where $\langle m\rangle_{i}$ is the average (over LSF) local magnetic moment in $\mu_{\mathrm{B}}$ of the $i$ th alloy component. In the EMTO-CPA and ELSGF calculations, the transverse magnetic disorder is modeled using the disordered local moment (DLM) model [68] for all alloy components (the longitudinal disorder on each site is effectively reduced to an average magnetic moment $\langle m\rangle_{i}$ in this case).

\section{Magnetic interactions and Curie temperature of $\mathrm{Cr}_{25} \mathrm{Fe}_{25} \mathrm{Co}_{25} \mathrm{Ni}_{25}$}

The Curie temperature is a characteristic of magnetic exchange interactions of the system and therefore, its modeling is important for the validation of the used theoretical approach. Here the Curie temperature of random fcc $\mathrm{Cr}_{25} \mathrm{Fe}_{25} \mathrm{Co}_{25} \mathrm{Ni}_{25}$ is calculated neglecting a local dependence of the magnetic exchange interactions [27] and using a classical Heisenberg Hamiltonian

$$
H=-\sum_{p} \sum_{i, j \in p} \sum_{\alpha, \beta=\mathrm{Cr}, \mathrm{Fe}, \mathrm{Co}, \mathrm{Ni}} J_{p}^{\alpha \beta} c_{i}^{\alpha} c_{j}^{\beta} \mathbf{e}_{i} \mathbf{e}_{j},
$$

where $J_{p}^{\alpha \beta}$ are the magnetic exchange interactions between $\alpha$ and $\beta$ alloy components at the $p$ th coordination shell, $\mathbf{e}_{i}$ is the direction of the spin at site $i$, and $c_{i}^{\alpha}$ takes on value 1 if site $i$ is occupied by atom $\alpha$ and 0 otherwise.

The magnetic exchange interactions were calculated using the magnetic force theorem [69-71] implemented in the Lyngby version of the EMTO-CPA code [67]. The calculations

TABLE III. On-site screening constants and average charges of atomic spheres in random fcc $\mathrm{Cr}_{25} \mathrm{Fe}_{25} \mathrm{Co}_{25} \mathrm{Ni}_{25}$. The DLM-LSF results are discussed below.

\begin{tabular}{lccrrr}
\hline \hline & \multicolumn{3}{c}{$T=0 \mathrm{~K}(\mathrm{FM}$ state) } & \multicolumn{2}{c}{$T=750 \mathrm{~K}$ (DLM-LSF) } \\
\cline { 2 - 5 } Element & $\alpha_{i}^{0}$ & $\left\langle q_{i}\right\rangle(\mathrm{ELSGF})$ & $\bar{q}_{i}(\mathrm{CPA})$ & $\alpha_{i}^{0}$ & $\left\langle q_{i}\right\rangle$ (ELSGF) \\
\hline $\mathrm{Cr}$ & 0.768 & -0.261 & -0.267 & 0.774 & -0.251 \\
$\mathrm{Fe}$ & 0.997 & -0.019 & -0.018 & 0.867 & -0.026 \\
$\mathrm{Co}$ & 0.744 & 0.100 & 0.104 & 0.748 & 0.097 \\
$\mathrm{Ni}$ & 0.806 & 0.180 & 0.181 & 0.800 & 0.179 \\
\hline \hline
\end{tabular}


TABLE IV. Average local magnetic moments (in $\mu_{\mathrm{B}}$ ) of alloy components in random fcc $\mathrm{Cr}_{25} \mathrm{Fe}_{25} \mathrm{Co}_{25} \mathrm{Ni}_{25}$. The DLM-LSF results are discussed below.

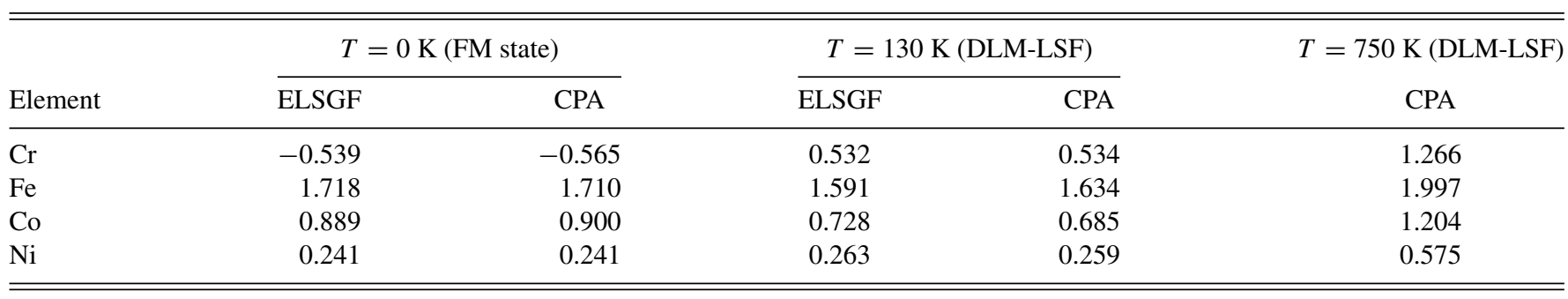

have been done for the Wigner-Seitz radius of 2.63 a.u. (or the fcc lattice constant of $3.561 \AA$ ), which was estimated to be the Wigner-Seitz radius at $130 \mathrm{~K}$ using the Debye-Grüneisen model [72,73] with the ELSGF total energies.

Since the system is essentially paramagnetic at the Curie temperature, the magnetic exchange interactions were calculated in the paramagnetic state with LSF at $130 \mathrm{~K}$ using the DLM local magnetic configuration (DLM-LSF) for every alloy component. In this way, the magnetic interactions also contain a contribution from LSF at the corresponding temperature.

In Fig. 8 the local magnetic moments of alloy components are shown in the paramagnetic (DLM-LSF) state as obtained in the ELSGF supercell calculations for fcc $\mathrm{Cr}_{25} \mathrm{Fe}_{25} \mathrm{Co}_{25} \mathrm{Ni}_{25}$. Comparing them with the results for the FM state in Ref. [27], one notices that they deviate substantially less from their average magnitude than local magnetic moments in the FM state. This is especially the case for $\mathrm{Cr}$, whose magnetic moment is mostly driven by LSF in the paramagnetic state. This means that the local magnetic moments in the paramagnetic state are much less sensitive to the chemical local environment of the corresponding atoms than in the FM state. Therefore, it is expected that magnetic exchange interactions should also be much less sensitive to the local environment effects in the paramagnetic state compared

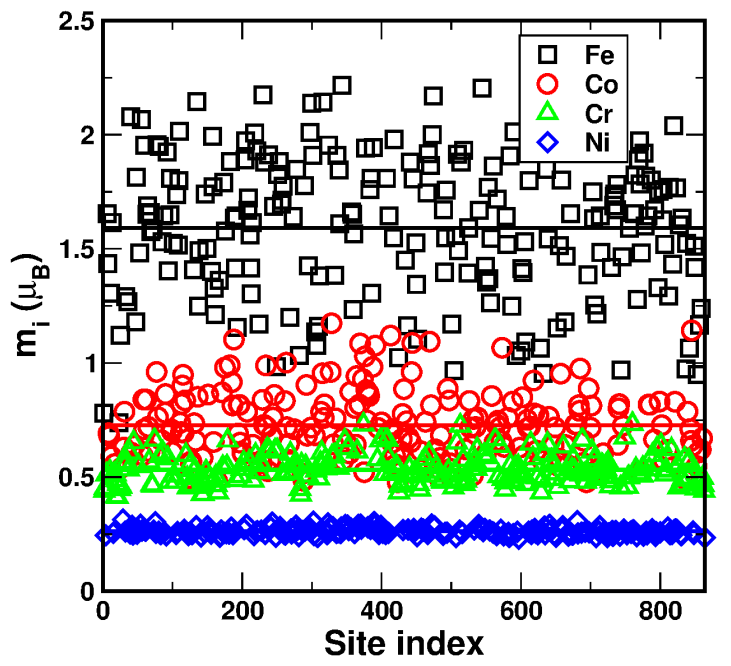

FIG. 8. Local magnetic moments of random fcc $\mathrm{Cr}_{25} \mathrm{Fe}_{25} \mathrm{Co}_{25} \mathrm{Ni}_{25}$ from the ELSGF DLM-LSF calculations at $130 \mathrm{~K}$ shown for all the atoms in the supercell using their site indexes. Thick lines show the average magnitudes for each alloy component. to those in the FM state [27], and the use of their average values in statistical thermodynamics modeling is a reasonable approximation.

In Table $\mathrm{V}$ the calculated magnetic exchange interactions at the first four coordination shells are shown. One notices that the magnetic exchange interactions at the first coordination shell are dominating. They are of the antiferromagnetic type if one of the alloy components is $\mathrm{Cr}$ and of the ferromagnetic type between $\mathrm{Fe}, \mathrm{Co}$, and $\mathrm{Ni}$, except for the $\mathrm{Fe}-\mathrm{Fe}$ interaction, which is small and antiferromagnetic. For the first coordination shell, the spatially averaged magnetic exchange interactions in the FM state obtained in the EMTO-CPA calculations are also shown. They are very similar to those in the DLM-LSF state. This means that dominating magnetic interactions depend little on the global magnetic state. Thus, the DLM-LSF interactions indeed provide a reasonable representation of the interactions in the FM state close to the Curie temperature.

The Heisenberg Monte Carlo simulations were done using a $12 \times 12 \times 12(\times 4)$ supercell with interactions presented in Table V. The calculated Curie temperature is about $120 \mathrm{~K}$, in good agreement with experimental data.

\section{E. Effective chemical interactions and ordering in $\mathrm{Cr}_{25} \mathrm{Fe}_{25} \mathrm{Co}_{25} \mathrm{Ni}_{25}$}

As in the case of the magnetic exchange interactions, the dependence of the chemical interactions on the local environment of atoms is disregarded. Also, the effects of thermal lattice vibrations are not accounted for. Nevertheless, the

TABLE V. Magnetic exchange interactions $J_{l m n}^{i j}$ (in mRy) in random fcc $\mathrm{Cr}_{25} \mathrm{Fe}_{25} \mathrm{Co}_{25} \mathrm{Ni}_{25}$ from EMTO-CPA calculations in the paramagnetic state with LSF at $130 \mathrm{~K}$. For comparison, the magnetic exchange interactions in the FM state are given in parentheses for the first coordination shell.

\begin{tabular}{lcrrr}
\hline \hline Pair $(i j)$ & $J_{110}^{i j}$ & \multicolumn{1}{c}{$J_{200}^{i j}$} & \multicolumn{1}{c}{$J_{211}^{i j}$} & \multicolumn{1}{c}{$J_{220}^{i j}$} \\
\hline Fe-Fe & $-0.0272(0.0893)$ & 0.2113 & -0.0493 & -0.0497 \\
Fe-Cr & $-0.4496(-0.3941)$ & 0.0877 & -0.0619 & 0.0556 \\
Fe-Co & $0.2270(0.3535)$ & 0.0430 & 0.0121 & -0.0658 \\
Fe-Ni & $0.1846(0.1295)$ & -0.0002 & 0.0235 & -0.0421 \\
Cr-Cr & $-0.1961(-0.1416)$ & -0.0197 & -0.0192 & 0.0297 \\
Cr-Co & $-0.1307(-0.1205)$ & 0.0532 & -0.0238 & 0.0114 \\
Cr-Ni & $-0.0056(-0.0012)$ & 0.0176 & -0.0045 & -0.0037 \\
Co-Co & $0.1887(0.2953)$ & -0.0109 & 0.0228 & -0.0444 \\
Co-Ni & $0.1037(0.0788)$ & -0.0074 & 0.0161 & -0.0208 \\
Ni-Ni & $0.0444(0.0179)$ & -0.0018 & 0.0074 & -0.0073 \\
\hline
\end{tabular}


TABLE VI. Effective charge transfer between alloy components $\left|\bar{q}_{i j}\right|$, intersite screening constant at the first coordination shell $\alpha_{1}^{i j}$, and effective pair interactions (in mRy) at the first four coordination shells. The interactions at the first coordination shell in parentheses are without electrostatic contribution [cf. Eq. (14)].

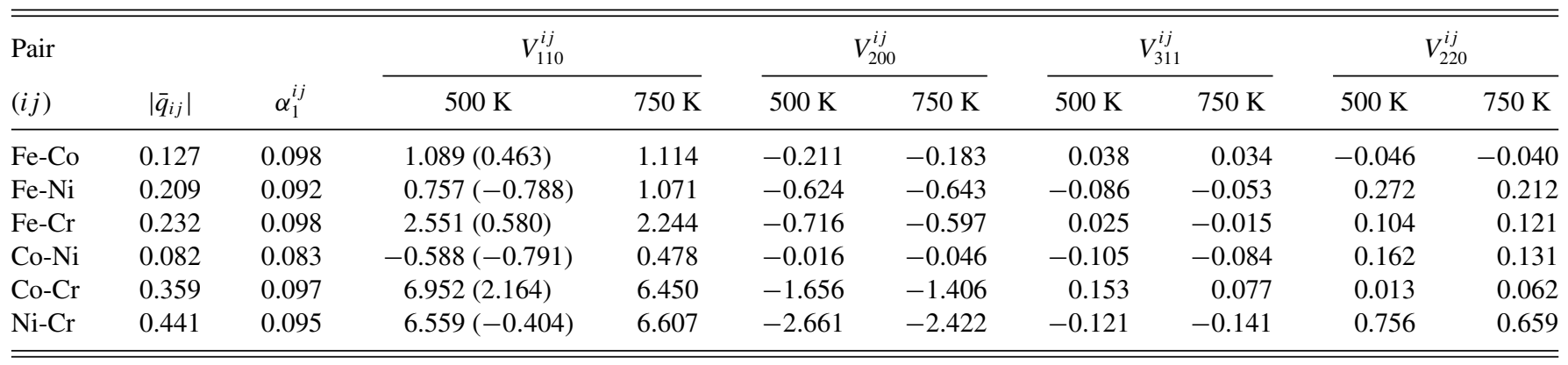

calculated effective interactions still depend on the temperature through the lattice constant and the magnetic state due to LSF. For statistical modeling of the atomic SRO at $720 \mathrm{~K}$, the effective chemical interactions were calculated by the SGPM method $[31,48,49]$ implemented in the Lyngby version of the EMTO-CPA code [67] in the DLM-LSF state at $750 \mathrm{~K}$ and a Wigner-Seitz radius of 2.68 a.u. (lattice constant $3.629 \AA$ ). Another set of interactions was also used [evaluated at $500 \mathrm{~K}$ and a Wigner-Seitz radius of 2.66 a.u. (lattice constant $3.602 \AA$ )] to estimate the temperature of a possible order-disorder phase transition as well as the temperature dependence of the effective interactions. The Wigner-Seitz radii at those temperatures were estimated in the Debye-Grüneisen model.

The SGPM pair interactions contain two contributions [49]

$$
V_{p}^{i j}=V_{p}^{i j-\mathrm{scr}}+V_{p}^{i j-1 \mathrm{e}},
$$

where $V_{p}^{i j-1 \mathrm{e}}$ is the one-electron contribution or GPM potential, and $V_{p}^{i j-s c r}$ is the screened Coulomb interaction at the $p$ th coordination shell for the $i-j$ pair of alloy components, which is given by Eq. (11). The effective charge transfer $\bar{q}_{i j}$ and intersite screening constants $\alpha_{p}^{i j}$ defining $V_{p}^{i j-\text { scr }}$ are shown in Table VI, together with the calculated effective pair interactions at the first four coordination shells for 500 and $750 \mathrm{~K}$.

The strongest interactions are between pairs of $\mathrm{Cr}$-Co and $\mathrm{Cr}-\mathrm{Ni}$ at the first coordination shell. They are positive and thus, according to Eq. (7), these nearest-neighbor pairs experience quite strong attractive interactions to each other. This is also the case for Cr-Fe pairs at the first coordination shell, but interaction is substantially weaker. One notices that these interactions tend to increase when lowering temperature, and besides, the $\mathrm{Cr}$-Co interaction becomes stronger than the Cr-Ni one.

The reason for the strong attraction between $\mathrm{Cr}$ and the other alloy components at the first coordination shell is the electrostatic interaction between these alloy components due to their size difference. This is clearly seen in Table VI, where the contribution from the one-electron term in Eq. (14) is separately given at the first coordination shell. One notices for instance, that without electrostatic contribution, the nearest-neighbor $\mathrm{Cr}-\mathrm{Ni}$ interaction is even negative.

The other interactions at the first coordination shell (between $\mathrm{Fe}, \mathrm{Co}$, and $\mathrm{Ni}$ ) are weak, and therefore, the main ordering is going to be between these three metals (Me) and
$\mathrm{Cr}$. Moreover, since the $\mathrm{Cr}-\mathrm{Me}$ interactions at the second coordination shell are also relatively strong and negative, the dominating order should be of the (001) type, with the formation of a kind of $\mathrm{L1}_{2}-(\mathrm{Fe}, \mathrm{Co}, \mathrm{Ni})_{3} \mathrm{Cr}$ alloy. As noted in the Introduction, energetic preference for this type of ordering was previously found in direct ab initio modeling in the FM state $[26,27]$.

In these papers [26,27], the ordering energy was estimated to be of the order of $-4.4 \mathrm{mRy} /$ atom (or $-0.24 \mathrm{eV} / \mathrm{cell}$ ). If one calculates the ordering energy of a $L 1_{2}-(\mathrm{Fe}, \mathrm{Co}, \mathrm{Ni})_{3} \mathrm{Cr}$ alloy in the FM state by the ELSGF method (using the 864atom supercells of random and partially ordered alloys for a fixed lattice constant of $3.588 \AA$ ), a value of $-4.2 \mathrm{mRy} /$ atom is obtained which is in agreement with the previous $a b$ initio calculations. However, the value is only $-2.0 \mathrm{mRy} /$ atom in the paramagnetic DLM-LSF state at $500 \mathrm{~K}$. Furthermore, the ordering energy of $\mathrm{L1}_{2}-(\mathrm{Fe}, \mathrm{Co}, \mathrm{Ni})_{3} \mathrm{Cr}$ obtained from the SGPM pair interactions at $500 \mathrm{~K}$ is $-2.7 \mathrm{mRy} /$ atom, and $-3.5 \mathrm{mRy} /$ atom in the FM state at $0 \mathrm{~K}$, in agreement with the direct total energy calculations. A large difference between ordering energies and effective interactions in different magnetic states was also observed in austenitic steels [19].

The size difference of alloy components is also the origin of the local lattice relaxations and strain-induced interactions which should be added to the effective chemical interactions obtained in the SGPM calculations. Unfortunately, the system is too complicated to determine such interactions accurately and reliably. Therefore, it is assumed that the strain-induced interactions mostly modify the nearest-neighbor $\mathrm{Ni}-\mathrm{Cr}$ and Co-Cr interactions by -1.0 and $-0.8 \mathrm{mRy}$, respectively [74]. Although the estimate is rather rough it should be sufficient to give a qualitative picture of ordering in this system, since these interactions are much smaller than the chemical ones.

Most of the multisite effective interactions are relatively weak. The strongest three-site interactions are found for the clusters connecting three nearest-neighbor sites along the [110] direction $V_{114}^{(3)-\alpha \beta \gamma[\delta]}$ (for the nomenclature of cluster indexes, see Ref. [24]), which are, for instance, $V_{114}^{(3)-\mathrm{NiNiNi}[\mathrm{Cr}]}=$ $-3.22 \mathrm{mRy}, V_{114}^{(3)-\mathrm{NiNiCo}[\mathrm{Cr}]}=V_{114}^{(3)-\mathrm{CoNiNi}[\mathrm{Cr}]}=-2.53 \mathrm{mRy}$, and $V_{114}^{(3)-\mathrm{NiCoNi}[\mathrm{Cr}]}=2.45 \mathrm{mRy}$. For other variations of alloy components, the interactions are significantly smaller, especially when containing Fe: $V_{114}^{(3)-\mathrm{FeFeFe}[\mathrm{Cr}]}=-0.44 \mathrm{mRy}$.

In the case of four-site interactions, the picture is a bit different. Again, the strongest interactions are connected with permutations of only $\mathrm{Ni}$ and $\mathrm{Cr}$, while the appearance of $\mathrm{Co}$ 


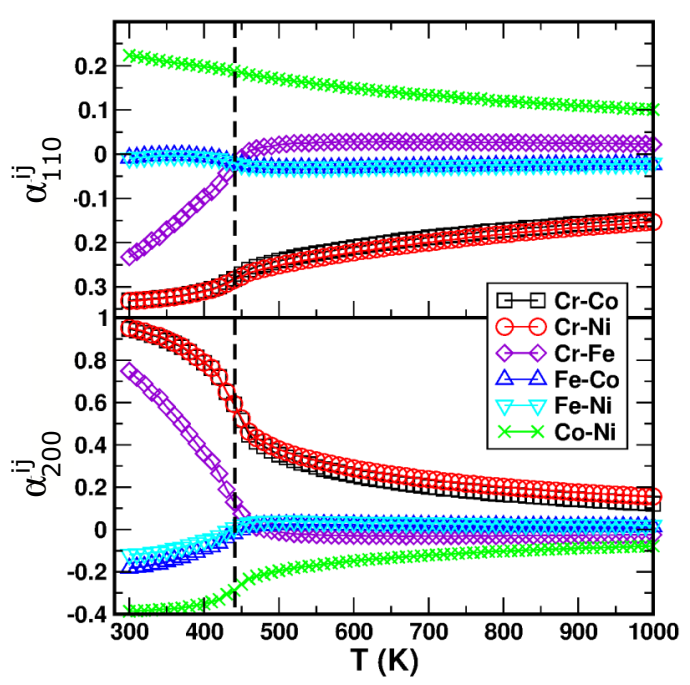

FIG. 9. Atomic SRO parameters $\alpha_{l m n}^{i j}$ at the first and second coordination shell from Monte Carlo simulations with the effective interactions at $750 \mathrm{~K}$. The dashed line shows the order-disorder transition.

substantially reduces the interaction. At the same time, the presence of Fe has only little effect. For instance, the strongest four-site interaction is $V_{111112}^{(4)-\mathrm{NiNiNiNi[Cr]}}=1.44 \mathrm{mRy}$ [this is for a tetrahedron formed by vectors $(1,1,0),(1,0,1)$, and $(2,0,0)$, in units of half of the fcc lattice constant], while, for instance, $V_{111112}^{(4)-\mathrm{CoCoCo}[\mathrm{Cr}]}=-0.27 \mathrm{mRy}$.

These multisite interactions are still smaller than the dominating pair interactions (actually, there are additional prefactors $\delta c_{i}^{\alpha}$ for multisite interactions in the Hamiltonian, which reduce their contribution significantly relative to pair interactions that are similar in magnitude). Thus, their contribution should not determine the type of ordering at elevated temperatures. Therefore, Monte Carlo simulations are only performed with the effective pair interactions corrected for the strain-induced interactions as described above. Interactions up to the 20th coordination shell were employed.

\section{F. Results of statistical simulations}

Monte Carlo simulations are done using two sets of interactions: for 750 and $500 \mathrm{~K}$. Figures 9 and 10 show the atomic SRO parameters $\alpha_{l m n}^{i j}$ as obtained in Monte Carlo simulations with effective interactions for both temperatures. They are very similar, with just small quantitative differences: For instance, as expected from the effective pair interactions, $\alpha_{110}^{\mathrm{NiCr}}$ is slightly more pronounced than $\alpha_{110}^{\mathrm{CoCr}}$ above the transition temperature with $750 \mathrm{~K}$ interactions. However, considering all the approximations used in this modeling, it is hard to say if this effect will also be present in more accurate theoretical simulations. A snapshot of $\mathrm{Cr}_{25} \mathrm{Fe}_{25} \mathrm{Co}_{25} \mathrm{Ni}_{25}$ at $700 \mathrm{~K}$ is shown in Fig. 11.

Although the effective interactions for 750 and $500 \mathrm{~K}$ are quite close to each other, they yield quite different orderdisorder transition temperatures: $420 \mathrm{~K}$ in the first case and $530 \mathrm{~K}$ in the second. Such a sensitivity of the ordering transition to the effective interactions means that the temperature dependence of the effective interactions is an important

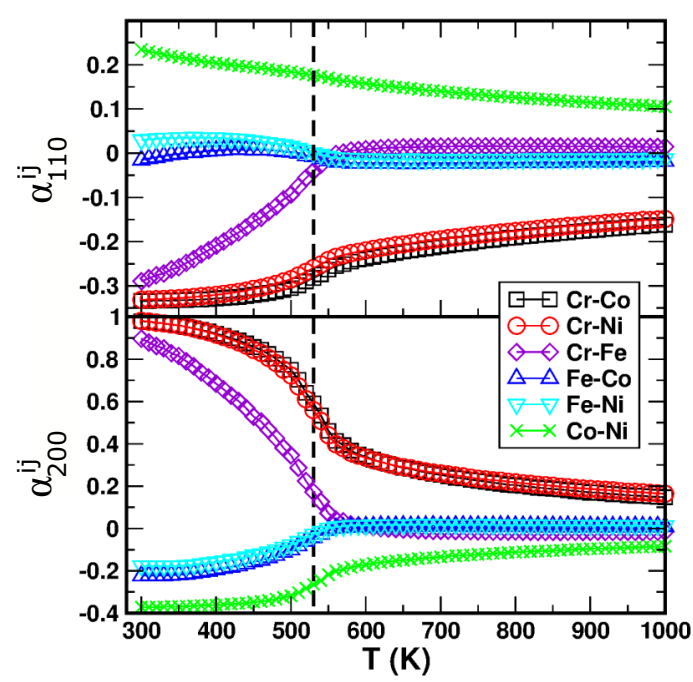

FIG. 10. Atomic SRO parameters $\alpha_{l m n}^{i j}$ at the first and second coordination shell from Monte Carlo simulations with the effective interactions at $500 \mathrm{~K}$. The dashed line shows the order-disorder transition.

feature, which should be considered in accurate statistical thermodynamics simulations.

A snapshot of the ordered structure just $30 \mathrm{~K}$ below the ordering phase transition is shown in Fig. 12. The ordered structure is indeed $\mathrm{L1}_{2}-(\mathrm{Fe}, \mathrm{Co}, \mathrm{Ni})_{3} \mathrm{Cr}$, with strong $\mathrm{Co}-\mathrm{Cr}$ and $\mathrm{Ni}-\mathrm{Cr}$ order reflected in the selected positioning of $\mathrm{Co}$ and $\mathrm{Ni}$ atoms with respect to $\mathrm{Cr}$ atoms. At the same time, $\mathrm{Fe}-\mathrm{Cr}$ atoms are almost uncorrelated at this temperature. The structure in Fig. 12 is purely hypothetical from a practical point of view. However, it shows the state in which the system tends to be and therefore, it is definitely relevant to what happens in this alloy at a local scale, even if long-range order cannot be established.

The calculated order-disorder transition temperature of about $500 \mathrm{~K}$ is quite low compared to the one where the timescale for equilibration is still within days. On the other hand, the effective interactions and the ordering energy are small to expect such a relatively high transition temperature if one believes in the strong stabilization role of the high configurational entropy of a multicomponent alloy. In fact, it is practically the same transition temperature as has been

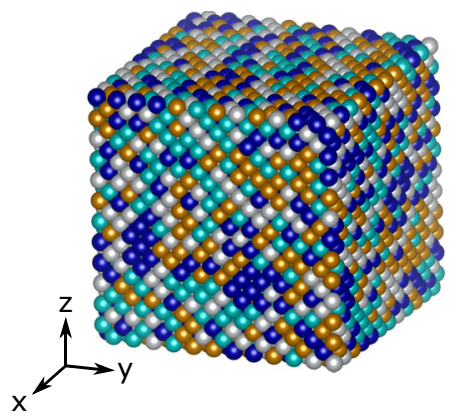

FIG. 11. A snapshot of a $12 \times 12 \times 12(\times 4)$ Monte Carlo simulation box at $700 \mathrm{~K}$ obtained with the $750 \mathrm{~K}$ interactions, $330 \mathrm{~K}$ above the order-disorder phase transition. $\mathrm{Cr}$ atoms are dark blue, $\mathrm{Co}$ light blue, Ni gray, and Fe yellow. 


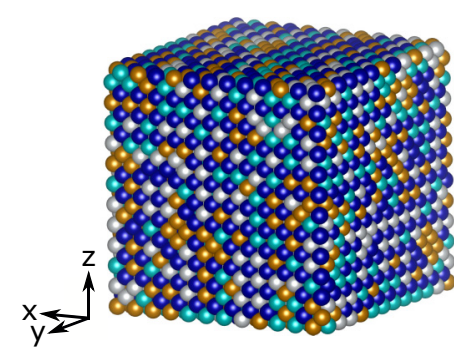

FIG. 12. A snapshot of a $12 \times 12 \times 12(\times 4)$ Monte Carlo simulation box at $500 \mathrm{~K}$ obtained with the $500 \mathrm{~K}$ interactions, just $30 \mathrm{~K}$ below the order-disorder phase transition. The colors of atoms are the same as in Fig. 11.

obtained in theoretical modeling of ordering of austenitic steels [19].

In order to see what happens, the configurational entropy has been determined from the heat capacity obtained in Monte Carlo simulations. Figure 13 shows that the deviation of the configurational entropy $S_{\text {conf }}$ due to atomic SRO from that of a four-component random alloy $\left(1.3863 k_{\mathrm{B}}\right)$ is relatively small down to the $\mathrm{L1}_{2}-(\mathrm{Fe}, \mathrm{Co}, \mathrm{Ni})_{3} \mathrm{Cr}$ ordering temperature. At lower temperatures, $S_{\text {conf }}$ is still relatively large and the decrease remains less than one expects for an $L 1_{2}$ ordered state of a binary $A-25$ at. $\% B$ alloy $\left(0.5623 k_{\mathrm{B}}\right)$. This change is only reached just above the next transition temperature. The explanation is clear: While the "corner" positions of the fcc unit cell become occupied almost exclusively by $\mathrm{Cr}$ atoms, there is essentially a random three-component alloy on the other three sublattices at the $L 1_{2}-(\mathrm{Fe}, \mathrm{Co}, \mathrm{Ni})_{3} \mathrm{Cr}$ ordering temperature. SRO ordering of $\mathrm{Fe}, \mathrm{Co}$, and $\mathrm{Ni}$ mainly takes place below this ordering temperature (see also the change in the $\alpha_{l m n}^{i j}$ with temperature in Fig. 10).

\section{G. Effect of atomic SRO and magnetic state on elastic constants}

To investigate the effect of atomic SRO and magnetic state on the elastic properties of $\mathrm{Cr}_{25} \mathrm{Fe}_{25} \mathrm{Co}_{25} \mathrm{Ni}_{25}$, ELSGF

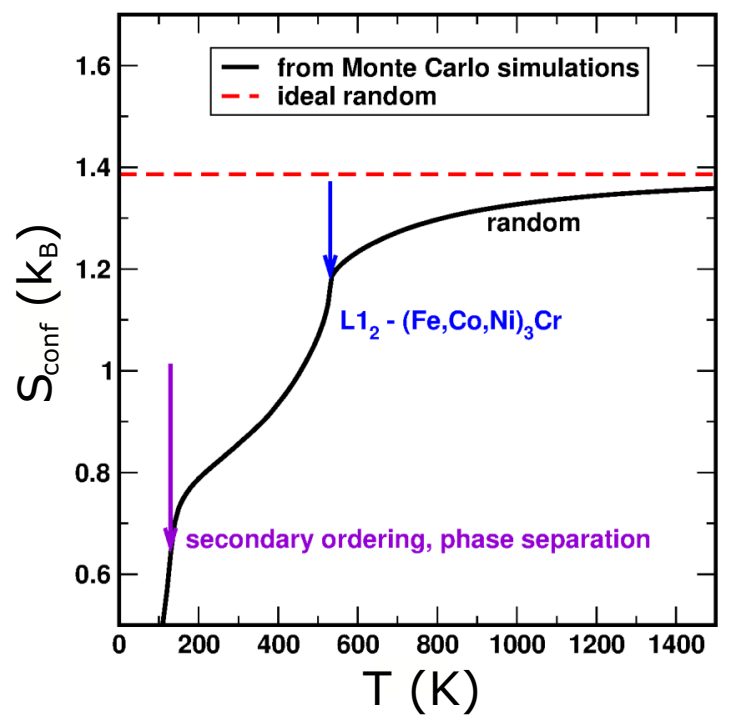

FIG. 13. Configurational entropy of $\mathrm{Cr}_{25} \mathrm{Fe}_{25} \mathrm{Co}_{25} \mathrm{Ni}_{25}$ from Monte Carlo simulations.
TABLE VII. Elastic constants $c_{i j}$, bulk modulus $B$, and shear modulus $c^{\prime}$ for (110) shears (in GPa), as calculated and from experiment (this work).

\begin{tabular}{lcccc}
\hline \hline & \multirow{2}{*}{$\begin{array}{c}\text { FM state at } 0 \mathrm{~K} \\
\text { random }\end{array}$} & \multicolumn{2}{c}{ DLM-LSF state at 300 K } & \\
\cline { 3 - 4 } & random & SRO at $T=1200 \mathrm{~K}$ & Experiment \\
\hline$c_{11}$ & 235 & 214 & 218 & $217(2)$ \\
$c_{12}$ & 157 & 136 & 134 & $138(2)$ \\
$c_{44}$ & 162 & 148 & 148 & $133(2)$ \\
$B$ & 183 & 162 & 162 & 164 \\
$c^{\prime}$ & 39 & 39 & 42 & 39 \\
\hline \hline
\end{tabular}

supercell calculations have been performed to determine the elastic constants $c_{i j}$ for a Wigner-Seitz radius of 2.64 a.u. (lattice parameter $3.575 \AA$ ). Supercells contained 512 atoms $(8 \times 8 \times 8)$ and atomic SRO parameters up to the eighth coordination shell were considered. For a supercell representing a random alloy, the atomic SRO parameters at the first three coordination shells were equal to zero, while for the supercell with atomic SRO, they were based on those obtained in Monte Carlo simulations with $750 \mathrm{~K}$ potentials at $1200 \mathrm{~K}$.

The most significant parameters are at the first two coordination shells for $\mathrm{Ni}-\mathrm{Cr}\left(\alpha_{110}^{\mathrm{NiCr}}=-0.130\right.$ and $\left.\alpha_{200}^{\mathrm{NiCr}}=0.120\right)$ and for Co-Cr pairs $\left(\alpha_{110}^{\mathrm{CoCr}}=-0.125\right.$ and $\left.\alpha_{200}^{\mathrm{CoCr}}=0.094\right)$. The other SRO parameters are much smaller in magnitude, including those for more distant coordination shells. Note that atomic correlation functions beyond the local interaction zone in the LSGF calculations practically do not contribute to the electronic structure and total energy. In the present calculations the local interaction zone was quite arbitrarily set to consist of two fcc coordination shells. However, the main goal of these particular calculations is to see the effect of just a relatively small amount of atomic SRO, which will be present in real alloys. Also two magnetic states were considered, the FM state and the DLM-LSF state at $300 \mathrm{~K}$ [75]. The results are presented in Table VII.

One notices that atomic SRO practically does not affect elastic properties in this alloy (small differences are within the accuracy of the whole theoretical methodology). This outcome is probably not surprising in view of experimental data for $\mathrm{Cu}-25$ at.\% $\mathrm{Au}$ and $\mathrm{Ni}-25$ at.\% Fe where even longrange order produces very little effect on the elastic properties $[76,77]$. It is also probably the reason why elastic constants (at least in this system) are not sensitive to the treatment of local environment effects in the first-principles calculations [75].

Both results, for alloys with and without atomic SRO, are in very good agreement with experimental data (as-grown state). At the same time, the elastic constants calculated in the FM state differ substantially from the DLM-LSF results. This shows the importance of magnetism for bonding in this system, like in the case of effective chemical interactions.

\section{H. SRO: Theory vs experiment}

The six partial SRO scattering patterns as obtained from electronic structure calculations at $720 \mathrm{~K}$, are shown in Fig. 14. Strong maxima at $X$ position are found for $\mathrm{Cr}-\mathrm{Co}$, $\mathrm{Cr}-\mathrm{Ni}$ and weaker ones for $\mathrm{Fe}-\mathrm{Co}, \mathrm{Fe}-\mathrm{Ni}$. A strong maximum 

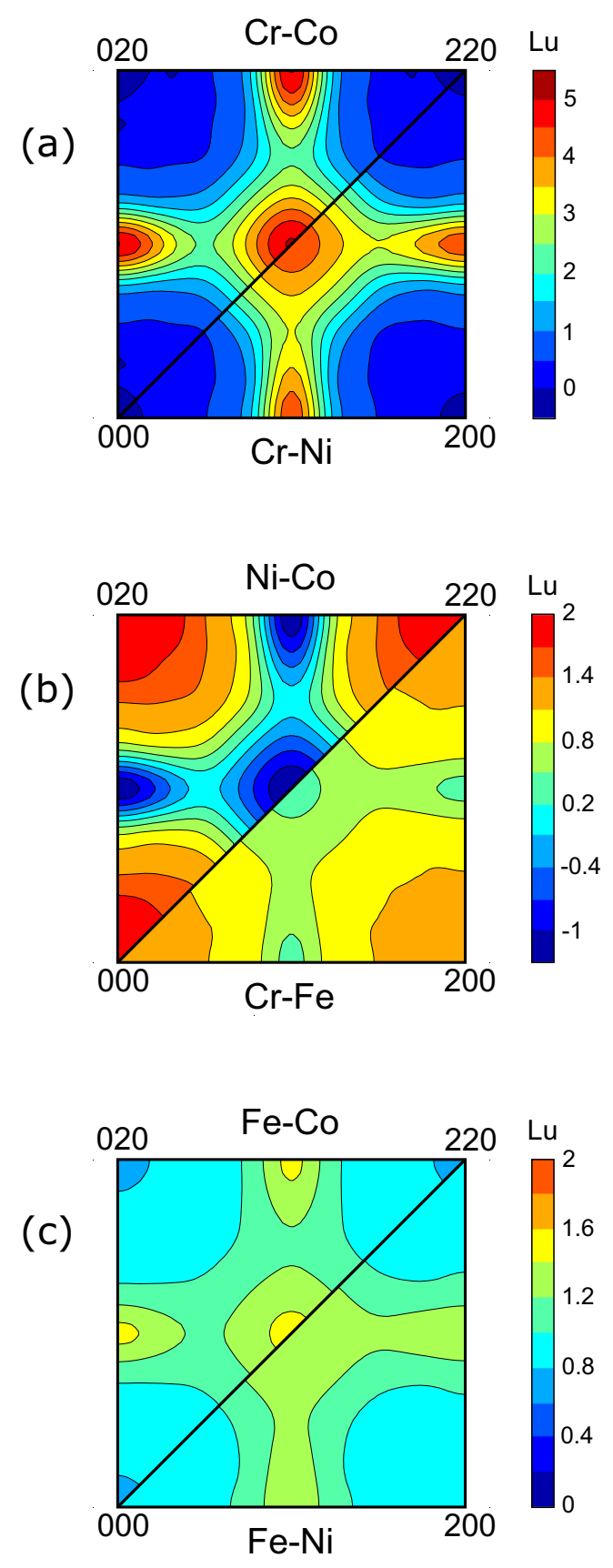

FIG. 14. SRO scattering at $\left(h_{x}, h_{y}, 0\right)$ positions for $720 \mathrm{~K}$ separately for the six partial SRO parameter sets $\alpha_{l m n}^{i j}$ as determined by the present electronic structure calculations.

at $\Gamma$ position is seen for Ni-Co. For $\mathrm{Cr}-\mathrm{Fe}$, weak modulations with a maximum not at a special position [at about $(0.3,0,0)$, not visible in Fig. 14] are present.

Figure 5 (top sectors) shows the elastic diffuse scattering one obtains from these electronic structure calculations for the scattering at the three edges. At the $\mathrm{Cr}$ edge, the largest scattering contrast is present for $\mathrm{Cr}-\mathrm{Ni}, \mathrm{Cr}-\mathrm{Co}$, and $\mathrm{Cr}-\mathrm{Fe}$, in this sequence (Table I). The partial SRO patterns of $\mathrm{Cr}-\mathrm{Ni}$ and Cr-Co exhibit a strong maximum at $X$ position (Fig. 14): More than $90 \%$ of the peak value [Fig. 5(a), top sector] comes from their partial SRO scattering. With 3.6 Lu the maximum is slightly higher than found experimentally ( 2.8 to $3.5 \mathrm{Lu})$.
Note that a rescaling of temperature (considering the $\alpha_{l m n}^{i j}$ at $820 \mathrm{~K}$ instead of $720 \mathrm{~K}$ ) already provides excellent agreement.

At the Fe edge the maximum at $X$ position does not dominantly come from $\mathrm{Fe}-\mathrm{Ni}$ and $\mathrm{Fe}-\mathrm{Co}$ with the highest scattering contrasts due to their weaker modulations (Fig. 14). Instead, $60 \%$ of the maximum at $X$ position [Fig. 5(b), top sector] comes from the partial $\mathrm{Cr}-\mathrm{Ni}$ and $\mathrm{Cr}-\mathrm{Co} \mathrm{SRO}$ scattering. With $2.0 \mathrm{Lu}$, the peak value closely fits the experimental findings (1.8 to $2.5 \mathrm{Lu}$ ).

At the Ni edge Ni-Co and Fe-Ni have the strongest scattering contrasts and are the reason for the slight intensity increase towards $\Gamma$ position [Fig. 5(c), top sector]. Ni-Cr with the third strongest (but already low) scattering contrast might be the reason for the weak maximum at $X$ position noted in experiment. Such a maximum, however, is not seen in the electronic structure calculations due to the large negative contribution from Ni-Co SRO scattering (Fig. 14).

The total SRO scattering as obtained from the electronic structure calculations at the three edges, was also analyzed using the SRO scattering within the $\left(h_{x}, h_{y}, 0\right)$ plane exclusively-like it was done to obtain $\alpha_{l m n}^{\text {eff }}$ from the elastic part of the diffuse x-ray scattering (Table II). This procedure offers the possibility to judge the quality in the outcome when no 3D data set but scattering only for the $\left(h_{x}, h_{y}, 0\right)$ plane is available. The effective SRO parameters $\alpha_{l m n}^{\text {eff }}$ then obtained from least-squares fitting, are given in Table II. One notes that $\alpha_{000}^{\mathrm{eff}}$ at the Cr edge is slightly larger than 1 , due to the large number of $X$ positions with high scattering within $\left(h_{x}, h_{y}, 0\right)$. The positive value of $\alpha_{110}^{\text {eff }}$ at the Ni edge confirms that local decomposition prevails.

The recalculated SRO scattering at the three edges is shown in Fig. 5 (left sectors). An excellent agreement in scattering between the directly calculated pattern and the recalculated pattern (using $\alpha_{l m n}^{\text {eff }}$ from Table II) is found. The reason for this agreement might be that the fitted patterns were free from experimental uncertainties. This outcome supports the validity to determine the leading $\alpha_{l m n}^{\text {eff }}$ just from slightly modulated scattering in the $\left(h_{x}, h_{y}, 0\right)$ plane.

\section{DISCUSSION}

A comparison between the SRO data from diffuse x-ray scattering and electronic structure calculations reveals a close similarity for the $\mathrm{Cr}$ edge and the $\mathrm{Fe}$ edge. In both cases, the maxima are located at $X$ position. Also, the effective SRO parameters $\alpha_{l m n}^{\text {eff }}$ in Table II are similar. The SRO data for the Ni edge, however, differ: On one side, they reveal a locally decomposed state, on the other side, a locally ordered state.

The weak scattering contrast and the large RRS for the Ni edge introduce higher uncertainties in evaluation than for the measurements at the $\mathrm{Cr}$ edge and the $\mathrm{Fe}$ edge. Also, on the basis of the calculated partial SRO parameter sets, the difference in SRO scattering between the maximum at about $(0.3,0,0)$ and the value at $(1,0,0)$ only amounts to $0.15 \mathrm{Lu}$. The different location of the SRO maximum might be related to the strong decomposition tendency introduced by the Ni-Co pair correlation: Without this partial SRO correlation, the maximum in total SRO scattering would be located at $X$ position.

If one compares the SRO data and the results from APT, an interesting feature is indicated: There is a deviation in the 
sequence of the field evaporation strength among the elements when pure and when within the alloy. This change suggests that $\mathrm{Cr}$ in $\mathrm{Cr}-\mathrm{Fe}-\mathrm{Co}-\mathrm{Ni}$ is preferential and not in a statistically uncorrelated way surrounded by $\mathrm{Ni}$ and $\mathrm{Co}$ elements. And just these pair correlations $\mathrm{Cr}-\mathrm{Ni}$ and $\mathrm{Cr}-\mathrm{Co}$ lead to the diffuse maximum at $X$ position.

The possible presence of strong atomic displacements was repeatedly considered for HEAs. Strong atomic displacements are known, e.g., for solid solutions of Al-rich Al-Cu (see Ref. [1] and references therein). They lead to a strong asymmetry in scattering around Bragg positions: here SRO scattering of a locally decomposed system has its maximum while the SE scattering contribution changes sign due to the sinus dependence in Eq. (5). In the present study, SE scattering causes a shift of the maximum in SRO scattering off $X$ position where SE scattering has zero intensity, too. As SE scattering has a linear dependence on $f_{i}-f_{j}$, a shift in direction may be achieved for alloys of neighbored elements when x-ray energies are tuned close to the absorption edges of both constituents ( $\mathrm{Ni}-\mathrm{Fe}, \mathrm{Ni}-\mathrm{Cr}, \mathrm{Fe}-\mathrm{Cr}$; for a summary, see Ref. [1]). Such a change in shift is indicated in the present study. As the magnitudes of the shifts are comparable between the binaries and the present quaternary, no stringent hint is provided for the presence of strong atomic displacements.

In contrast to the present study where APT showed that a single-phase state is present, there are two TEM studies where two-phase states were found for $\mathrm{Cr}-\mathrm{Fe}-\mathrm{Co}-\mathrm{Ni}[17,78]$. In both cases, however, no states of thermal equilibrium were studied: According to the present resistivity measurements (Fig. 1), neither aging at $1573 \mathrm{~K}$ for 1 week followed by water quench as reported by Kozak et al. [17] nor the as-cast state reported by Guo et al. [78] will result in equilibrium states. There are further indications that nonequilibrium states were studied. Kozak et al. [17] stated that an onset of phase decomposition seems to be indicated by the appearance of a heterogeneous morphology with small fully coherent regions ( $\leqslant 20 \mathrm{~nm}$ in size) with different composition. Guo et al. [78] noted a second phase at grain boundaries of their polycrystalline material, showing an enrichment in $\mathrm{Cr}$. As $\mathrm{Cr}$ has the highest diffusion coefficient in $\mathrm{Cr}-\mathrm{Mn}-\mathrm{Fe}-\mathrm{Co}-\mathrm{Ni}$ as well as in $\mathrm{Cr}-\mathrm{Fe}-\mathrm{Co}-\mathrm{Ni}[79,80]$, kinetics will be the reason.

\section{CONCLUSIONS}

As HEAs were often considered to possess microstructural features deviating from those in binary or ternary systems, it seems instructive to follow the location of the diffuse maxima from various binary solid solutions over the ternary $\mathrm{Fe}-21$ at.\% Cr-23 at.\% $\mathrm{Ni}$ [6] to the present quaternary CrFe-Co-Ni. (i) For Ni-Cr [20-23] it goes from $W$ over $X$ to $X$ position. (ii) For Fe-Cr [81] it changes from $\Gamma$ over $W$ to $\Gamma$ position. (iii) For Fe-Ni [82-84] it goes from $X$ over $\Gamma$ to $X$ position. The finding of local decomposition due to the Ni-Co pair correlation function is interesting and calls for a diffuse scattering experiment in the fcc solid solution which extends over the whole range of composition for temperatures above the allotropic transformation of $\mathrm{Co}$. In conclusion, no special features are seen in the SRO data of this multicomponent alloy.

For more detailed information of local order in $\mathrm{Cr}-\mathrm{Fe}-\mathrm{Co}-$ $\mathrm{Ni}$, a mosaic crystal spectrometer [85] would be required (besides 3D data sets) to improve data quality: Resonant Raman scattering $K-M_{\mathrm{II}} M_{\mathrm{III}}$ as well as Compton scattering could then be removed experimentally. As in the case of the SRO parameters, only effective linear displacement parameters would be accessible. However, as pair displacements are species dependent and have vector property (this yields 27 parameters per $l m n$ shell for a quaternary alloy), this is beyond the scope of a diffuse scattering study.

Equimolar $\mathrm{Cr}-\mathrm{Fe}-\mathrm{Co}-\mathrm{Ni}$ exhibits a pronounced ordering tendency towards the $L 1_{2}-(\mathrm{Fe}, \mathrm{Co}, \mathrm{Ni})_{3} \mathrm{Cr}$ structure, which can be stabilized at about $500 \mathrm{~K}$ according to a theoretical estimate. The strongest interactions occur between $\mathrm{Cr}-\mathrm{Co}$ and $\mathrm{Cr}-\mathrm{Ni}$ nearest-neighbor pairs and are mainly due to the electrostatic effects caused by the size difference of these atoms. The ordering tendency is much stronger in the FM state. However, the Curie temperature is quite low (about $120-130 \mathrm{~K}$ ), atoms are then almost immobile, and thus, it is impossible to follow the ordering transition experimentally.

It is demonstrated that a "high" configurational entropy by itself does not warrant stabilization of the random configurational state. Due to specific ordering between alloy components, equimolar $\mathrm{Cr}-\mathrm{Fe}-\mathrm{Co}-\mathrm{Ni}$ behaves actually like a quasibinary $\mathrm{Me}_{75} \mathrm{Cr}_{25}$ alloy with $L 1_{2}$ type of ordering. In fact, if one uses hypothetical Me-Cr interactions taken just as the average pair $\mathrm{Fe}-\mathrm{Cr}, \mathrm{Co}-\mathrm{Cr}$, and $\mathrm{Ni}-\mathrm{Cr}$ effective interactions at $500 \mathrm{~K}$, the order-disorder transition is $630 \mathrm{~K}$, i.e., just $100 \mathrm{~K}$ above the transition in the equimolar $\mathrm{Cr}-\mathrm{Fe}-\mathrm{Co}-\mathrm{Ni}$ alloy. The reason is simple: in this kind of ordering only one sublattice is occupied by preferentially $\mathrm{Cr}$ atoms, while there is a random alloy of the remaining alloy components on the three other fcc sublattices: High configurational entropy only leads to a partially random state.

The strong impact of the magnetic state on bonding in this alloy is also reflected in the sensitivity of the elastic constants of $\mathrm{Cr}_{25} \mathrm{Fe}_{25} \mathrm{Co}_{25} \mathrm{Ni}_{25}$ to the magnetic state as has been demonstrated in the first-principles calculations. At the same time, elastic constants seem to be practically unaffected by the atomic SRO, at least when its degree is not that large. It should be stressed that it does not mean that other alloy properties do not depend on atomic SRO. As has been observed experimentally, resistivity is apparently quite sensitive to the state of order. This point needs further theoretical investigation which is beyond the scope of the present work.

One puzzle remains unresolved: first-principles calculations at $0 \mathrm{~K}$ predict that random $\mathrm{Cr}_{25} \mathrm{Fe}_{25} \mathrm{Co}_{25} \mathrm{Ni}_{25}$ is unstable in the fcc structure, while experimentally it is fcc though the configurational state in this case can be different from random. Thermal magnetic and vibrational excitations can also stabilize the fcc structure at finite temperatures. The accurate theoretical investigation of this problem is hardly possible nowadays.

\section{ACKNOWLEDGMENTS}

The authors are grateful to E. Fischer for growing the single crystal. They gratefully acknowledge the European Synchrotron Radiation Facility (ESRF) for provision of synchrotron radiation at beamline ID01. B.S. thanks KAUST for providing measuring time for the APT studies. A.V.R. acknowledges the support of the Swedish Research Council 
(VR project 2015-05538), a European Research Council grant, the VINNEX center Hero-m, financed by the Swedish Governmental Agency for Innovation Systems (VINNOVA), Swedish industry, and the Royal Institute of Technology (KTH). Calculations were done using NSC (Linköping) and PDC (Stockholm) resources provided by the Swedish National Infrastructure for Computing (SNIC). A.V.R. and O.E.P. also acknowledge the financial support under the scope of the COMET program within the K2 Center "Integrated Computational Material, Process and Product Engineering (IC-MPPE)" (Project No 859480). This program is supported by the Austrian Federal Ministries for Transport, Innovation and Technology (BMVIT) and for Digital and Economic Affairs (BMDW), represented by the Austrian research funding association (FFG), and the federal states of Styria, Upper Austria and Tyrol.
[1] B. Schönfeld, Prog. Mater. Sci. 44, 435 (1999).

[2] W. Schweika, Disordered Alloys: Diffuse Scattering and Monte Carlo Simulation, Springer Tracts in Modern Physics No. 141 (Springer, Berlin, 1998).

[3] G. E. Ice and C. J. Sparks, Annu. Rev. Mater. Sci. 29, 25 (1999).

[4] V. M. Nield and D. A. Keen, Diffuse Neutron Scattering from Crystalline Materials (Clarendon, Oxford, 2001).

[5] G. Kostorz, in Physical Metallurgy, edited by D. E. Laughlin and K. Hono (Elsevier, Amsterdam, 2014), p. 1227.

[6] P. Cenedese, F. Bley, and S. Lefebvre, Acta Crystallogr. Sect. A 40, 228 (1984).

[7] S. Hashimoto and H. Iwasaki, Radiat. Eff. Defects Solids 124, 147 (1992).

[8] D. R. Haeffner and J. B. Cohen, Acta Metall. Mater. 40, 831 (1992).

[9] B. D. Butler and J. B. Cohen, Acta Metall. Mater. 41, 41 (1993).

[10] D. L. Larson, T. J. Prosa, R. M. Ulfig, B. P. Geiser, and T. F. Kelly, Local Electrode Atom Probe Tomography (Springer, New York, 2013).

[11] M. K. Miller and R. G. Forbes, Atom-Probe Tomography: The Local Electrode Atom Probe (Springer, New York, 2014).

[12] T. Boll, T. Al-Kassab, Y. Yuan, and Z. G. Liu, Ultramicroscopy 107, 796 (2007); see also T. Boll, Dissertation, University Göttingen, 2010.

[13] R. K. W. Marceau, A. V. Ceguerra, A. J. Breen, M. Palm, F. Stein, S. P. Ringer, and D. Raabe, Intermetallics 64, 23 (2015).

[14] Y. Zhang, T. T. Zuo, Z. Tang, M. C. Gao, K. A. Dahmen, P. K. Liaw, and Z. P. Lu, Prog. Mater. Sci. 61, 1 (2014).

[15] E. J. Pickering and N. G. Jones, Int. Mater. Rev. 61, 183 (2016).

[16] D. B. Miracle and O. N. Senkov, Acta Mater. 122, 448 (2017).

[17] R. Kozak, A. Sologubenko, and W. Steurer, Z. Kristallogr. Cryst. Mater. 230, 55 (2015).

[18] M. S. Lucas, G. B. Wilks, L. Mauger, J. A. Muñoz, O. N. Senkov, E. Michel, J. Horwath, S. L. Semiatin, M. B. Stone, D. L. Abernathy, and E. Karapetrova, Appl. Phys. Lett. 100, 251907 (2012).

[19] A. V. Ruban and M. Dehghani, Phys. Rev. B 94, 104111 (2016).

[20] W. Schweika and H. G. Haubold, Phys. Rev. B 37, 9240 (1988).

[21] B. Schönfeld, L. Reinhard, G. Kostorz, and W. Bührer, Phys. Status Solidi B 148, 457 (1988).

[22] B. Schönfeld, G. E. Ice, C. J. Sparks, H.-G. Haubold, W. Schweika, and L. B. Shaffer, Phys. Status Solidi B 183, 79 (1994).

[23] R. Caudron, M. Sarfati, M. Barrachin, A. Finel, F. Ducastelle, and F. Solal, J. Phys. I (France) 2, 1145 (1992).

[24] M. Rahaman, B. Johansson, and A. V. Ruban, Phys. Rev. B 89, 064103 (2014).
[25] P. Singh, A. V. Smirnov, and D. D. Johnson, Phys. Rev. B 91, 224204 (2015).

[26] C. Niu, A. J. Zaddach, A. A. Oni, X. Sang, J. W. Hurt, J. M. LeBeau, C. C. Koch, and D. L. Irving, Appl. Phys. Lett. 106, 161906 (2015).

[27] T. Fukushima, H. Katayama-Yoshida, K. Sato, M. Ogura, R. Zeller, and P. H. Dederichs, J. Phys. Soc. Jpn. 86, 114704 (2017).

[28] D. de Fontaine, J. Appl. Crystallogr. 4, 15 (1971).

[29] T. B. Wu, E. Matsubara, and J. B. Cohen, J. Appl. Crystallogr. 16, 407 (1983).

[30] S. Hashimoto, Acta Crystallogr. Sect. A 43, 481 (1987).

[31] F. Ducastelle, Order and Phase Stability in Alloys (NorthHolland, Amsterdam, 1991).

[32] B. Borie and C. J. Sparks Jr., Acta Crystallogr. Sect. A 27, 198 (1971).

[33] P. Georgopoulos and J. B. Cohen, J. Phys. (Paris), Colloq. 38, C7-191 (1977).

[34] A. V. Ruban and H. L. Skriver, Phys. Rev. B 55, 856 (1997).

[35] In its turn, the quasibinary pair interactions are $\widetilde{V}_{p}^{(2)-\alpha \delta}=$ $V_{p}^{(2)-\alpha \alpha[\delta]}$ and $\tilde{V}_{p}^{(2)-\alpha \beta}=V_{p}^{(2)-\alpha \alpha[\delta]}+V_{p}^{(2)-\beta \beta[\delta]}-2 V_{p}^{(2)-\alpha \beta[\delta]}$.

[36] E. Prince, Ed., International Tables for Crystallography (International Union of Crystallography, Chester, 2006), Vol. C.

[37] S. Sasaki, KEK Report No. 88-14 (Nat. Lab. High Energy Physics, Tsukuba, Japan, 1989).

[38] O. K. Andersen, O. Jepsen, and G. Krier, in Lectures on Methods of Electronic Structure Calculations, edited by V. Kumar, O. K. Andersen, and A. Mookerjee (World Scientific, Singapore, 1994), p. 63.

[39] L. Vitos, Computational Quantum Mechanics for Materials Engineers (Springer, London, 2007).

[40] P. E. Blöchl, Phys. Rev. B 50, 17953 (1994).

[41] G. Kresse and D. Joubert, Phys. Rev. B 59, 1758 (1999).

[42] P. Soven, Phys. Rev. 156, 809 (1967); B. L. Gyorffy, Phys. Rev. B 5, 2382 (1972).

[43] L. Vitos, I. A. Abrikosov, and B. Johansson, Phys. Rev. Lett. 87, 156401 (2001).

[44] I. A. Abrikosov, A. M. N. Niklasson, S. I. Simak, B. Johansson, A. V. Ruban, and H. L. Skriver, Phys. Rev. Lett. 76, 4203 (1996); I. A. Abrikosov, S. I. Simak, B. Johansson, A. V. Ruban, and H. L. Skriver, Phys. Rev. B 56, 9319 (1997).

[45] O. E. Peil, A. V. Ruban, and B. Johansson, Phys. Rev. B 85, 165140 (2012).

[46] The Lyngby version of the EMTO code properly takes into consideration electrostatics in random alloys in contrast to other existing versions. It is distributed by A. V. Ruban. 
[47] A. V. Ruban and H. L. Skriver, Phys. Rev. B 66, 024201 (2002); A. V. Ruban, S. I. Simak, P. A. Korzhavyi, and H. L. Skriver, ibid. 66, 024202 (2002).

[48] F. Ducastelle and F. Gautier, J. Phys. F: Met. Phys. 6, 2039 (1976).

[49] A. V. Ruban, S. Shallcross, S. I. Simak, and H. L. Skriver, Phys. Rev. B 70, 125115 (2004).

[50] J. P. Perdew and Y. Wang, Phys. Rev. B 45, 13244 (1992).

[51] J. P. Perdew, K. Burke, and M. Ernzerhof, Phys. Rev. Lett. 77, 3865 (1996).

[52] H. J. Monkhorst and J. D. Pack, Phys. Rev. B 13, 5188 (1976).

[53] G. Kresse and J. Hafner, Phys. Rev. B 47, 558 (1993).

[54] G. Kresse and J. Hafner, Phys. Rev. B 49, 14251 (1994).

[55] G. Kresse and J. Furthmüller, Phys. Rev. B 54, 11169 (1996).

[56] T. Boll, Z.-Y. Zhu, T. Al-Kassab, and U. Schwingenschlögl, Microsc. Microanal. 18, 964 (2012).

[57] T. Boll and T. Al-Kassab, Ultramicroscopy 124, 1 (2013).

[58] F. Biggs, L. B. Mendelsohn, and J. B. Mann, At. Data Nucl. Data Tables 16, 201 (1975).

[59] W. A. Reed and P. Eisenberger, Phys. Rev. B 6, 4596 (1972).

[60] B. E. Warren, X-Ray Diffraction (Dover, New York 1990).

[61] Y. H. Zhang, Y. Zhuang, A. Hu, J. J. Kai, and C. T. Liu, Scr. Mater. 130, 96 (2017).

[62] S. Zhao, G. M. Stocks, and Y. Zhang, Acta Mater. 134, 334 (2017).

[63] The stacking fault energy in the fcc structure is roughly equal to the double energy difference of the fcc and hcp structures at the same volume.

[64] Y.-F. Kao, S.-K. Chen, T.-J. Chen, P.-C. Chu, J.-W. Yeh, and S.-J. Lin, J. Alloys Compd. 509, 1607 (2011).

[65] M. S. Lucas, D. Belyea, C. Bauer, N. Bryant, E. Michel, Z. Turgut, S. O. Leontsev, J. Horwath, S. L. Semiatin, M. E. McHenry, and C. W. Miller, J. Appl. Phys. 113, 17A923 (2013).

[66] A. V. Ruban, A. B. Belonoshko, and N. V. Skorodumova, Phys. Rev. B 87, 014405 (2013).

[67] A. V. Ruban, Phys. Rev. B 95, 174432 (2017).

[68] In the DLM approach, the paramagnetic state is modeled by a random alloy of spin-up and spin-down magnetic moment orientations of every component of the system. For details see B. L. Gyorffy, A. J. Pindor, J. Staunton, G. M. Stocks, and H. Winter, J. Phys. F: Met. Phys. 15, 1337 (1985).
[69] T. Oguchi, K. Terakura, and N. Hamada, J. Phys. F: Met. Phys. 13, 145 (1983); T. Oguchi, K. Terakura, and A. R. Williams, Phys. Rev. B 28, 6443 (1983).

[70] A. I. Liechtenstein, M. I. Katsnelson, and V. A. Gubanov, J. Phys. F: Met. Phys. 14, L125 (1984).

[71] A. I. Liechtenstein, M. I. Katsnelson, V. P. Antropov, and V. A. Gubanov, J. Magn. Magn. Mater. 67, 65 (1987).

[72] V. L. Moruzzi, J. F. Janak, and K. Schwarz, Phys. Rev. B 37, 790 (1988).

[73] P. A. Korzhavyi, A. V. Ruban, S. I. Simak, and Yu. Kh. Vekilov, Phys. Rev. B 49, 14229 (1994).

[74] These interactions yield a local relaxation energy of about $9 \mathrm{meV} /$ atom, which is in reasonable agreement with the PAW results for the FM state $(6.6 \mathrm{meV} /$ atom $)$.

[75] In order to check local environment effects, the elastic properties were also calculated by the EMTO-CPA method for random alloys in both magnetic states. However, they were practically the same as the ELSGF results, where such effects are taken into consideration, which means that they are not important in this system.

[76] P. A. Flinn, G. M. McManus, and J. A. Rayne, J. Phys. Chem. Solids 15, 189 (1960).

[77] P. Turchi, Y. Calvayrac, and F. Plicque, Phys. Status Solidi A 45, 229 (1978).

[78] S. Guo, C. Ng, Z. Wang, and C. T. Liu, J. Alloys Compd. 583, 410 (2014).

[79] K.-Y. Tsai, M.-H. Tsai, and J.-W. Yeh, Acta Mater. 61, 4887 (2013).

[80] W. Kucza, J. Dabrowa, G. Cieślak, K. Berent, T. Kulik, and M. Danielewski, J. Alloys Compd. 731, 920 (2018).

[81] L. Reinhard, J. L. Robertson, S. C. Moss, G. E. Ice, P. Zschack, and C. J. Sparks, Phys. Rev. B 45, 2662 (1992).

[82] S. Lefebvre, F. Bley, M. Bessiere, M. Fayard, M. Roth, and J. B. Cohen, Acta Crystallogr. Sect. A 36, 1 (1980).

[83] S. Lefebvre, F. Bley, M. Fayard, and M. Roth, Acta Metall. 29, 749 (1981).

[84] X. Jiang, G. E. Ice, C. J. Sparks, L. Robertson, and P. Zschack, Phys. Rev. B 54, 3211 (1996).

[85] G. E. Ice and C. J. Sparks, Nucl. Instrum. Methods Phys. Res., Sect. A 291, 110 (1990). 